\title{
Genotype-phenotype associations in French patients with phenylketonuria and importance of genotype for full assessment of tetrahydrobiopterin responsiveness
}

Elise Jeannesson-Thivisol ${ }^{1,2}$, François Feillet ${ }^{1,2+}$, Céline Chéry ${ }^{1,2}$, Pascal Perrin ${ }^{1,2}$, Shyue-Fang Battaglia-Hsu ${ }^{1,2}$, Bernard Herbeth ${ }^{1,2}$, Aline Cano ${ }^{3}$, Magalie Barth" ${ }^{4}$, Alain Fouilhoux ${ }^{5}$, Karine Mention ${ }^{6}$, François Labarthe ${ }^{7}$, Jean-Baptiste Arnoux ${ }^{8}$, François Maillot ${ }^{9}$, Catherine Lenaerts ${ }^{10}$, Cécile Dumesnil $^{11}$, Kathy Wagner ${ }^{12}$, Daniel Terral ${ }^{13}$, Pierre Broué ${ }^{14}$, Loïc de Parscau ${ }^{15}$, Claire Gay ${ }^{16}$, Alice Kuster ${ }^{17}$, Antoine Bédu ${ }^{18}$, Gérard Besson ${ }^{19}$, Delphine Lamireau ${ }^{20}$, Sylvie Odent ${ }^{21}$, Alice Masurel ${ }^{22}$, Jean-Louis Guéant ${ }^{1,2+}$ and Fares Namour ${ }^{1,2^{*}+}$

\begin{abstract}
Background: Mutations in Phenylalanine Hydroxylase $(P A H)$ gene cause phenylketonuria. Sapropterin (BH4), the enzyme cofactor, is an important therapeutical strategy in phenylketonuria. However, PAH is a highly polymorphic gene and it is difficult to identify BH4-responsive genotypes. We seek here to improve prediction of $\mathrm{BH} 4-$ responsiveness through comparison of genotypes, BH4-loading test, predictions of responsiveness according to the literature and types and locations of mutations.
\end{abstract}

Methods: A total of 364 French patients among which, $9 \%$ had mild hyperphenylalaninemia, $17.7 \%$ mild phenylketonuria and $73.1 \%$ classical phenylketonuria, benefited from a 24-hour BH4-loading test and had the PAH gene sequenced and analyzed by Multiplex Ligation Probe Amplification.

Results: Overall, $31.6 \%$ of patients were BH4-responsive. The number of different mutations found was 127 , including 26 new mutations. The mutations c.434A > T, c.500A > T, c.529G > C, c.1045 T> G and c.1196 T>C were newly classified as being BH4-responsive. We identified 261 genotypes, among which 46 were newly recognized as being $\mathrm{BH} 4$-responsive. Even though patients carry 2 responsive alleles, BH4-responsiveness cannot be predicted with certainty unless they present mild hyperphenylalaninemia. BH4-responsiveness cannot be predicted in patients carrying one responsive mutation only. In general, the milder the phenotype is, the stronger the BH4-response is. Almost exclusively missense mutations, particularly in exons 12,11 and 8 , are associated with $\mathrm{BH} 4$-responsiveness and any other type of mutation predicts a negative response.

Conclusions: This study is the first of its kind, in a French population, to identify the phenotype associated with several combinations of PAH mutations. As others, it highlights the necessity of performing simultaneously BH4 loading test and molecular analysis in monitoring phenylketonuria patients.

Keywords: Phenylketonuria, Tetrahydrobiopterin, BH4-loading test, Genotype, BH4-responsiveness

\footnotetext{
* Correspondence: bernard.namour@univ-lorraine.fr

${ }^{\dagger}$ Equal contributors

${ }^{1}$ Reference Center for Inherited Metabolic Diseases, University Hospital of Nancy, 9 ave Forêt de Haye, BP 184, 54511 Vandoeuvre-lès-Nancy, France ${ }^{2}$ INSERM U954, Department of Nutrition-Genetics-Environmental Risk Exposure, University of Lorraine, 9 ave Forêt de Haye, BP 184, 54511 Vandoeuvre-lès-Nancy, France

Full list of author information is available at the end of the article
}

\section{$\int$ Biomed Central}

(c) 2015 Jeannesson-Thivisol et al. Open Access This article is distributed under the terms of the Creative Commons Attribution 4.0 International License (http://creativecommons.org/licenses/by/4.0/), which permits unrestricted use, distribution, and reproduction in any medium, provided you give appropriate credit to the original author(s) and the source, provide a link to the Creative Commons license, and indicate if changes were made. The Creative Commons Public Domain Dedication waiver (http://creativecommons.org/publicdomain/zero/1.0/) applies to the data made available in this article, unless otherwise stated. 


\section{Background}

Phenylketonuria (PKU, OMIM 261600) is an autosomal recessive disorder caused by a deficient hepatic phenylalanine hydroxylase (PAH) activity [1]. To date, no less than 890 disease causing mutations have been registered to the BioPKU database [2]. In general, the genotype dictates the phenotype [3]. Individuals with simultaneous presence of two null alleles exhibit the classic PKU (cPKU) phenotype, whereas individuals with mutations bearing residual enzyme activity display the mild PKU (mPKU) or the mild hyperphenylalaninemia (mHP).

$\mathrm{PAH}$ is a homotetrameric or homodimeric enzyme, whose activity is regulated by the binding of its substrate, Phenylalanine (Phe), and its cofactor, tetrahydrobiopterin (BH4). Each subunit of this multimeric enzyme is composed of the regulatory, the catalytic and the oligomerization domains [4].

The mainstay of the treatment for PKU remains the low-phenylalanine diet [5]. Additional strategy involving the use of sapropterin, a specific pharmacological chaperone similar to the physiologic cofactor tetrahydrobiopterin, is being introduced [6]. Sapropterin enhances PAH enzymatic activity, through multiple mechanisms including (1) stabilization of $\mathrm{PAH}$ dimer/tetramer, (2) up-regulation of gene expression, and (3) stabilization of mRNA $[4,7,8]$. However, sapropterin is not efficient in all patients. The degree of sapropterin-responsiveness depends on the presence of responsive mutations [9] having still residual PAH activity [10]. Since PAH is a multimeric protein, it depends also on the allelic complementation of the particular genotype $[11,12]$.

Numerous studies have investigated mutations in the $P A H$ gene as well as prediction of $\mathrm{BH} 4$-responsiveness based on genotypes. However, only few have additionally performed BH4-loading tests [13-20]. Thus the status of several genotypes is unknown and whether they are $\mathrm{BH} 4$-responsive or not needs to be clarified.

We have performed a systematical research of mutations in French PKU patients and systematically evaluated the response to oral BH4-loading test. The aim was to improve the prediction of $\mathrm{BH} 4$-responsiveness by relating genetic information such as type, location and combination of mutations, to $\mathrm{BH} 4$-loading test responses.

\section{Methods}

\section{Patients}

A total of 364 hyperphenylalaninemic patients, including 42 siblings, were investigated. They represented mixed origin people from 20 French Centers for Inborn Errors of Metabolism. According to their blood Phenylalanine concentration at time of diagnosis, these patients were assigned to one of the three phenotype categories: mild hyperphenylalaninemia (mHP, $180<$ Phe $<600 \mu \mathrm{mol} / \mathrm{L}$ ); mild phenylketonuria (mPKU, $600<$ Phe $<1200 \mu \mathrm{mol} / \mathrm{L}$ ) and classical phenylketonuria (cPKU, Phe $>1200 \mu \mathrm{mol} / \mathrm{L}$ ) [21].

\section{BH4-responsiveness}

The BH4 oral loading test protocol has been standardized and published in the French High Health Authority (HAS) 2010 recommendations [22]. Patients were considered as BH4-responsive if an oral bolus of $\mathrm{BH} 4$ dose (20 mg/kg body weight) lowered blood Phe concentration by at least $30 \%$ at one time point within 24 hours. In $35 \%$ of patients, BH4-loading test was performed during the neonatal period, in $42 \%$ between 1 month and 18 years and in $23 \%$ during adulthood.

\section{DNA isolation, PCR amplification, dHPLC (denatured High Performance Liquid Chromatography) and sequencing} Informed consent for genotype assessment was obtained from patients (or parents) for being included in the study. Genotyping analyses were conducted in a single laboratory. Genomic DNA was extracted from blood samples using the Nucleon BACC3 Genomic DNA Extraction Kit (GE Healthcare). PCR was carried out with 13 sets of oligonucleotides amplifying all 13 exons of the gene and the exon-intron junctions. Amplicons were separated by dHPLC as previously reported [23]. Fragments showing an abnormal dHPLC pattern were purified (Qiaquick PCR purification kit, Qiagen), quantified and sequenced using the ABI PRISM Big Dye terminator V1.1 sequencing kit and the 3100-Avant Genetic Analyzer (Applied Biosystems). When no mutation was identified with dHPLC, all exons were sequenced.

\section{Multiplex Ligation-dependent Probe Amplification (MLPA) analysis}

When only one mutation was found, MLPA analysis was performed with the SALSA MLPA kit P055 (MRC) in order to detect large deletions or duplications.

\section{Variants classification}

A mutation was considered as novel if not listed in the NCBI database [24] and hence was submitted to this database. An unclassified mutation was considered as $\mathrm{BH} 4$-responsive when $\mathrm{BH} 4$ challenging was positive and the genotype was either homozygous for this mutation or compound heterozygous combining this mutation with a null mutation $[10,12]$. A null mutation is a nonsense mutation or a mutation yielding a protein with less than $1 \%$ predicted residual activity (PRA). If a homozygous genotype was found to be associated with unresponsiveness in at least five patients, the mutated allele was considered as non-responsive. In addition, a genotype was newly recognized as responsive if 1 ) there was at least one responsive patient in the present study and 2) it was not 
previously described at the BioPKU database. All genotypes have been submitted to the BioPKU database [2].

\section{Molecular modeling}

Missense mutations newly described as BH4-responsive were modeled by in silico analysis using Pymol molecular visualization system and the published structure of truncated PAH with Protein Data Bank entry 1MMK (catalytic domain and dimerization motif).

Secondary structure was drawn with Dog 2.0 software using information from Erlandsen and colleagues [25], UniprotKB/Swissprot (P00439) and NCBI Reference sequence NP_000268.1.

\section{Statistical analysis}

Statistical analysis was performed by using the SAS software package version 9.3 (SAS Institute). Genotypes, mutations and related descriptive characteristics were presented as frequency, mean and/or percentage. Associations were tested using $t$-test, Chi-square $(\mathrm{Chi}-2)$ or Fisher's exact tests. Tests were judged significant at $\mathrm{p} \leq 0.05$.

\section{Results}

\section{Phenotype distribution}

Thirty-three patients were identified as mHP (9\%), 65 as mPKU (17.7\%) and 266 as cPKU (73.1\%). Patients with siblings similarly affected by PKU appeared to share the same phenotype. 24 hours after BH4-loading approximately one third of patients (31.6\%) experienced a reduction in their Phe concentration of at least $30 \%$. Overall, more responders were found in groups with the less severe phenotypes, with the positive response rate at $90.9 \%$ for mHP, $69.2 \%$ for mPKU and $14.7 \%$ for cPKU, respectively $(p<0.0001$; Fisher exact test) (Additional file 1: Figure $\mathrm{S} 1$ ). In addition, the milder the phenotype is, the higher the response rate is. Indeed, $71 \%$ of $\mathrm{mHP}$ with a positive test versus $37 \%$ of cPKU with a positive test presented with a responsive rate higher than $50 \%$.

$\mathrm{mHP}$ patients $\mathrm{BH} 4$ responders were tested at initial Phe concentrations at the start of BH4-testing (H0) ranking between 114 and $990 \mu \mathrm{mol} / \mathrm{L}($ mean = 366). Three patients among 33 with $\mathrm{mHP}$ were $\mathrm{BH} 4$ nonresponsive (c.[1045 T $>\mathrm{C}] ;[1243 \mathrm{G}>\mathrm{A}] \quad$ (p. [Ser349Pro]; [Asp415Asn]); c. [734 T > C]; [1315+1G >A] (p. [Val245Ala]; [?]) and c. [261C > A]; [1065 + 1G > A] (p. [Ser87Arg]; [?]) genotypes). Two of them were tested between 3 and 18 yo, both at initial Phe concentration of $144 \mu \mathrm{mol} / \mathrm{L}$, lower that minimum concentration required for testing. For PKU, there was no difference in Phe concentration at the start of $\mathrm{BH} 4$ testing between responsive aPKU $(n=37 ; \mathrm{mH} 0=600 \mu \mathrm{mol} / \mathrm{L} \quad(216-$ 1116) $)$ and non-responsive aPKU $(n=15 ; \quad \mathrm{mH} 0=$ $672 \mu \mathrm{mol} / \mathrm{L}(318-1026))$ neither between responsive cPKU $(n=29 ; \mathrm{mH0}=1206 \mu \mathrm{mol} / \mathrm{L}(342-2160))$ and non- responsive cPKU $(n=170 ; \quad \mathrm{mH} 0=1374 \mu \mathrm{mol} / \mathrm{L} \quad(300$ 4320)) (T-test; $\mathrm{p}$ values $=0.28$ and 0.22 respectively) .

\section{Mutation detection rate and mutations spectrum}

No mutation was found for one classical PKU patient and only a single heterozygous pathogenic mutation could be identified in one mHP, $2 \mathrm{mPKU}$ and $2 \mathrm{cPKU}$. Twelve deletions and one duplication were found by MLPA. Mutation detection rate was $97.3 \%$ with sequencing alone and $99.05 \%$ when combined with MLPA.

A total of 127 different mutations were detected among which 26 new ones. Mutations were mapped to the catalytic (63.8\%), regulatory (17.3\%) and oligomerization (2.4\%) domains and $15.7 \%$ localized in intronic regions. One deletion spanned across catalytic and oligomerization domains. Mutations types were as follows: $59.8 \%$ missense, $15.7 \%$ splice-site, $9.4 \%$ frameshift small deletions or insertions, $8.7 \%$ nonsense mutations, as well as $3.9 \%$ large deletions, $1.6 \%$ in-frame small deletions or duplications and $0.8 \%$ large duplications.

Sixty-one mutations were private mutations. The most frequent mutation found was c.1066-11G > A (8.6\%). Some of the most frequent alleles (frequency $>1 \%$ ) were significantly associated with the severity of the clinical phenotype like c.1066-11G > A (PRA 0) and c.1055delG (p.Gly352Valfs*48) (Fisher exact test, $p<0.05$ ). On the other hand, c.1241A > G (p.Tyr414Cys), c.1169A > G (p. Glu390Gly), c.1208C > T (p.Ala403Val), c.204A > T (p.Arg68Ser) and c.898G > T (p.Ala300Ser), known to have PRA $>30 \%$, were associated with moderate phenotypes (mHP and mPKU, Fisher exact test, $p<0.001$ ). Although predicted residual activity of c.782G $>$ A (p.Arg261Gln) is 38.5, this mutation was found to be more frequent in the cPKU group (Fisher exact test, $p<0.05$ ).

\section{Mutations responsiveness to $\mathrm{BH} 4$}

Data available in the literature indicated that among the 127 pathogenic mutations we found, 28 are $\mathrm{BH} 4$ responsive and $17 \mathrm{BH} 4$-unresponsive. Among the 82 left mutations, we succeeded in classifying 5 as BH4responsive and 7 as $\mathrm{BH} 4$-unresponsive while the 70 others remained with an unknown status. The new mutation c.1196 T > C (p.Val399Ala) was considered BH4-responsive since after $\mathrm{BH} 4$-loading test (initial Phe concentration $1020 \mu \mathrm{mol} / \mathrm{L})$, a $97 \%$ decrease in Phe concentration was observed in a compound heterozygous patient carrying this mutation with c.1066-11G > A mutation generating a protein with negligible residual activity. Similar conclusion was drawn for the c.1045 T > G mutation (p.Ser349Ala) since positive BH4-responses were observed in one homozygous mPKU patient and another compound heterozygous patient carrying an additional non-responsive allele. The mutations c.434A $>\mathrm{T}$ (p.Asp145Val), c.500A > T 
(p.Asn167Ile) and c.529G > C (p.Val177Leu) were also classified as $\mathrm{BH} 4$-responsive since they were found in compound heterozygous patients carrying a second null mutation. These mutations were mapped to the catalytic domain of the protein and are shown on a modeled tridimensional structure representing the PAH protein complexed with $\mathrm{BH} 4$, thienylalanine (analog substrate) and iron (Additional file 2: Figure S2 and Additional file 3: Figure S3). Another group of five unclassified mutations fitted the criteria to be classified as responsive mutations: c.266_267insC (p.Ala90Cfs*12), c.1249 T > A (p.Tyr417Asn), c.887A > G (p.Asp296Gly newly described mutation), c.1199+1G >C and c.493G > A (p.Ala165Thr) but where classified as unclear especially because they were observed in patients with a BH4responsive rate comprised between 30 and 40 percent (see Discussion). Thus c.[266_267insC]; [842C > T] genotype was observed in one patient displaying a $36 \%$ decrease in Phe concentration (H0 $1560 \mu \mathrm{mol} / \mathrm{L}$; tested between 3 and 10 yo) and c. [1249 T > A]; [1315 + 1G > A] genotype was observed in one mPKU with a $37 \%$ decrease in Phe concentration (H0 $702 \mu \mathrm{mol} / \mathrm{L}$; neonatal test). The c.887A > G (p.Asp296Gly) mutation was found in one patient combined with the non-responsive c.1222C > T mutation (p. Arg408Trp). This patient showed a $36 \%$ decrease in Phe concentration (H0 $1326 \mu \mathrm{mol} / \mathrm{L}$; tested between 10 and 18 yo), however p.Arg408Trp mutation is proposed to have a dominating negative effect [20]. One cPKU patient with c. $[1199+1 G>C] ;[1066-11 G>A]$ genotype showed a $37.5 \%$ Phe concentration decrease (H0 $1152 \mu \mathrm{mol} / \mathrm{L}$; tested between 10 and 18 yo) but 1 mPKU ((H0 $822 \mu \mathrm{mol} / \mathrm{L}$; tested between 3 and 10 yo) and $1 \mathrm{cPKU}$ (H0 $3258 \mu \mathrm{mol} / \mathrm{L}$; tested as neonate) with the same genotype were non-responsive. In addition, one homozygote c. $[1199+1 \mathrm{G}>\mathrm{C}] ;[1199+1 \mathrm{G}>\mathrm{C}]$ cPKU were non-responsive ( $\mathrm{H} 01842 \mu \mathrm{mol} / \mathrm{L}$; tested at adulthood). Finally, c. [493G > A]; [493G > A] genotype was observed in two cPKU siblings. The one tested between 3 and 10 years of age showed no decrease in Phe concentration $(\mathrm{H} 0384 \mu \mathrm{mol} / \mathrm{L})$ while the one tested in the neonatal period showed a $36 \%$ decrease ( $\mathrm{H0} 1680 \mu \mathrm{mol} / \mathrm{L})$ but is not currently under $\mathrm{BH} 4$ treatment.

Conversely, 6 nonsense mutations - coding for p.Glu57*, p.Glu66*, p.Tyr168*; p.Tyr216*, p.Asp222*, p.Glu280*, and the deletion c.1055delG (p.Gly352Vfs*48), observed in 7 homozygous patients with no response to $\mathrm{BH} 4$ challenging, were considered as $\mathrm{BH} 4$-unresponsive.

\section{Genotype-phenotype associations}

In Table 1, the BH4-loading tests for the 261 tabulated genotypes are listed together with the relative frequency and associated PKU phenotypes. Most of the genotypes were associated with a unique class of PKU severity. However, c. [194 T > C]; [1208C > T] (p.[Ile65Thr]; [Ala403Val], $n=3)$ and c. $[1208 \mathrm{C}>\mathrm{T}] ;$ [1222C > T] (p.[Ala403Val]; [Arg408Trp], $n=2)$ genotypes were associated with both $\mathrm{mHP}$ and $\mathrm{mPKU}$ and 12 other genotypes $(n=36)$ were observed in both mPKU and cPKU. In addition, c. [1223G > A]; [1241A > G] (p.[Arg408Gln]; [Tyr414Cys], $n=2$ ) was inconsistently associated with $\mathrm{mHP}$ and $\mathrm{cPKU}$.

The most frequent genotype $(n=11,4.2 \%)$ was c. [838G > A]; [838G > A] (p.[Glu280Lys]; [Glu280Lys]). Overall, 21 genotypes had a frequency higher than $1 \%$ $(n \geq 3)$. Among them, the observed phenotype was consistent with the predicted phenotype (according to the predictive residual activity) for the severe mutations only. Indeed, individuals with a combination of two mutations with low PRA $(<2)$ were consistently associated with either mPKU or most often with cPKU. Three genotypes, c. [782G > A]; [782G > A] (p.[Arg261Gln]; [Arg261Gln], $n=8), \quad$ c. $[194 \mathrm{~T}>\mathrm{C}] ; \quad[782 \mathrm{G}>\mathrm{A}] \quad$ (p. [Ile65Thr]; [Arg261Gln], $n=3$ ) and c. [143 T $>$ C]; [143 T > C] (p. [Leu48Ser]; [Leu48Ser], $n=3$ ), were associated to severe $\mathrm{cPKU}$ or mPKU phenotypes despite high residual activity for both mutations (p.Arg261Gln: PRA 38.5; p.Ile65Thr: PRA 25.3; p.Leu48Ser: PRA 39). In addition, in genotypes combining a mild mutation (PRA > 20) and a severe mutation (PRA $<2)$, mild mutation did not always dominate. For example, c. [782G > A]; [1315 + $1 \mathrm{G}>\mathrm{A}]$ (p. [Arg261Gln]; [?], $n=5), \quad$ c. $[1222 \mathrm{C}>\mathrm{T}]$; [1241A > G] (p. [Arg408Trp]; [Tyr414Cys], $n=5), \quad$ c. [194 T > C]; [1066-11G > A] (p.[Ile65Thr]; [?], $n=4)$ and c. [782G > A]; [1066-11G > A] (p.[Arg261Gln]; [?], $n=4$ ) were all associated with either cPKU or mPKU.

A total of $36.7 \%$ of all genotypes included at least one $\mathrm{BH} 4$-responsive patient. 83 genotypes displayed a response rate higher than $40 \%$ and 9 genotypes displayed a response between $30 \%$ and $40 \%$. This includes 46 genotypes newly described as BH4-responsive. Among all patients, 215 displayed a genotype consisting of two alleles whose $\mathrm{BH} 4$-responsiveness was reported in the literature. Among those, 51 were carriers of 2 alleles previously expected to be BH4-responsive. However, only $66.7 \%(34 / 51)$ responded positively to the BH4-loading test. Indeed, $100 \%$ of mHP patients carrying 2 BH4responsive alleles responded to $\mathrm{BH} 4$ administration but only $85.7 \%$ of mPKU and $42.3 \%$ of cPKU patients (Additional file 4: Figure S4). The presence of a single responsive mutation predicted a high rate of responsiveness in $\mathrm{mHP}(84.6 \%)$ and $\mathrm{mPKU}(73.1 \%)$ patients, while only $13.5 \%$ of cPKU patients carrying one responsive mutation and one non-responsive mutation exhibited positive BH4-loading test (Additional file 4: Figure S4). Another 50 of the 215 patients whose $\mathrm{BH} 4$-responsiveness could be predicted according to the literature, carried two nonresponsive alleles. In these patients, the observed phenotype matched the predicted phenotype in all but two (Additional file 4: Figure S4), who experienced decrease, 
Table 1 Genotypes observed in French patients with phenylketonuria. Association with clinical phenotypes and response to BH4 loading-test

\begin{tabular}{|c|c|c|c|c|c|c|c|c|c|c|c|c|c|}
\hline \multirow[t]{2}{*}{$\begin{array}{l}\text { Genotypes (?: neither } \\
\text { mutation found with } \\
\text { sequencing nor } \\
\text { rearrangement } \\
\text { found in MLPA; } \\
\text { \#: newly described } \\
\text { mutation) }\end{array}$} & & \multirow[t]{2}{*}{$\begin{array}{l}\text { Number } \\
\text { (freq \%) }\end{array}$} & \multicolumn{3}{|c|}{$\begin{array}{l}\text { Number of BH4- } \\
\text { loading tests } \\
\text { (responders/non } \\
\text { responders) }\end{array}$} & \multirow[t]{2}{*}{$\begin{array}{l}\text { Total BH4 } \\
\text { loading test } \\
\text { responders }\end{array}$} & \multirow[t]{2}{*}{$\begin{array}{l}\text { Total BH4 } \\
\text { loading } \\
\text { test non- } \\
\text { responders }\end{array}$} & \multirow[t]{2}{*}{$\begin{array}{l}\text { Number of responsive } \\
\text { and/or non-responsive } \\
\text { patients previously } \\
\text { recorded in the BioPKU } \\
\text { database for each } \\
\text { responsive genotype } \\
\text { of the present study }\end{array}$} & \multicolumn{3}{|c|}{$\begin{array}{l}\text { Predicted BH4- } \\
\text { sensitivity (PRA) } \\
\text { according to the } \\
\text { literature ([10] } \\
\text { except }{ }^{\mathrm{a}}[9] ; \\
\text { b }[30] ; \text { c.1066- } \\
11 \mathrm{G}>\text { A [2]) }\end{array}$} & \multicolumn{2}{|c|}{$\begin{array}{l}\text { Responsiveness } \\
\text { as proposed in } \\
\text { this manuscript } \\
\text { if "Predicted } \\
\text { BH4-sensitivity } \\
\text { according to the } \\
\text { literature" = unk }\end{array}$} \\
\hline & & & $\mathrm{mHP}$ & $\mathrm{mPKU}$ & $\mathrm{CPKU}$ & & & & mut1 & mut2 & mut3 & mut1 & mut2 \\
\hline c. $[1 \mathrm{~A}>\mathrm{G}] ;[782 \mathrm{G}>\mathrm{A}]$ & p. [Met1Val]; [Arg261GIn] & $1(0.38)$ & - & - & $\begin{array}{l}\text { 1) }(0 / \\
1)\end{array}$ & 0 & 1 (cPKU) & & unk & $\begin{array}{l}\mathrm{R}^{\mathrm{a}} \\
(38.5)\end{array}$ & - & & \\
\hline c. $[60 \mathrm{G}>\mathrm{C}] ;[473 \mathrm{G}>\mathrm{A}]$ & $\begin{array}{l}\text { p. [GIn20His]; } \\
\text { [Arg158GIn] }\end{array}$ & $1(0.38)$ & - & - & $\begin{array}{l}1(0 / \\
1)\end{array}$ & 0 & 1 (cPKU) & & unk & $\mathrm{R}(10)$ & - & & \\
\hline $\begin{array}{l}\text { c. }[60+5 G>A]^{\#} ;[1042 C> \\
G]\end{array}$ & p. [?]; [Leu348Val] & $1(0.38)$ & - & - & $\begin{array}{l}1(0 / \\
1)\end{array}$ & 0 & 1 (cPKU) & & unk & $\mathrm{R}(41)$ & - & & \\
\hline $\begin{array}{l}\text { c. }\left[115 \_117 \text { delTTC]; }\right. \\
{[1042 C>\mathrm{G}]}\end{array}$ & $\begin{array}{l}\text { p. [Phe39del]; } \\
\text { [Leu348Val] }\end{array}$ & $1(0.38)$ & - & - & $\begin{array}{l}\text { 1) }(0 / \\
1)\end{array}$ & 0 & $1(\mathrm{cPKU})$ & & $\mathrm{R}(20)$ & $R(41)$ & - & & \\
\hline $\begin{array}{l}\text { c. [115_117delTTC; }[1315+ \\
\text { 1G>A] }\end{array}$ & p. [Phe39del]; [?] & $2(0.77)$ & - & - & $\begin{array}{l}2(0 / \\
2)\end{array}$ & 0 & 2 (2 cPKU) & & $\mathrm{R}(20)$ & $N R(0)$ & - & & \\
\hline c. $[117 \mathrm{C}>\mathrm{G}] ;[\mathrm{i} 85 \mathrm{~T}>\mathrm{G}]^{\#}$ & $\begin{array}{l}\text { p. [Phe39Leu]; } \\
\text { Val262Gly] }\end{array}$ & $1(0.38)$ & - & - & $\begin{array}{l}1(0 / \\
1)\end{array}$ & 0 & 1 (cPKU) & & $\mathrm{R}(49)$ & unk & - & & \\
\hline c. $[117 \mathrm{C}>\mathrm{G}] ;[814 \mathrm{G}>\mathrm{T}]$ & p. [Phe39Leu]; [Gly272*] & $1(0.38)$ & - & - & $\begin{array}{l}\text { 1) }(0 / \\
1)\end{array}$ & 0 & 1 (cPKU) & & R (49) & NR (1) & - & & \\
\hline c. $[117 C>G] ;[1169 A>G]$ & $\begin{array}{l}\text { p. [Phe39Leu]; } \\
\text { [Glu390Gly] }\end{array}$ & $1(0.38)$ & - & - & $\begin{array}{l}1(1 / \\
0)\end{array}$ & $1(\mathrm{cPKU})$ & 0 & no data & R (49) & $\begin{array}{l}\mathrm{R} \\
(72.5)\end{array}$ & - & & \\
\hline c. [143 T>C]; [143 T>C] & p. [Leu48Ser]; [Leu48Ser] & $3(1.15)$ & - & $\begin{array}{l}2(2 / \\
0)\end{array}$ & $\begin{array}{l}1(1 / \\
0)\end{array}$ & $\begin{array}{l}3(2 \mathrm{mPKU} \\
1 \mathrm{cPKU})\end{array}$ & 0 & $37 \mathrm{R} ; 5 \mathrm{NR}$ & R (39) & R (39) & - & & \\
\hline c. [143 T > C]; [165delT] & $\begin{array}{l}\text { p. [Leu48Ser]; } \\
\text { [Phe55Leufs*6] }\end{array}$ & $2(0.77)$ & - & - & $\begin{array}{l}2(2 / \\
0)\end{array}$ & $2(\mathrm{cPKU})$ & 0 & 2 PKU R & R (39) & unk & - & & \\
\hline c. $[143 \mathrm{~T}>\mathrm{C}] ;[526 \mathrm{C}>\mathrm{T}]$ & p. [Leu48Ser]; [Arg176*] & $1(0.38)$ & - & - & $\begin{array}{l}1(1 / \\
0)\end{array}$ & 1 (cPKU) & 0 & no data & R (39) & NR (1) & - & & \\
\hline $\begin{array}{l}\text { c. [143 T > C]; } \\
\text { [547_548delinsTT] }\end{array}$ & $\begin{array}{l}\text { p. [Leu48Ser]; } \\
\text { [Glu183Leu] }\end{array}$ & $1(0.38)$ & - & - & $\begin{array}{l}\text { 1)/ } \\
\text { 1) }\end{array}$ & 0 & $1(\mathrm{cPKU})$ & & R (39) & unk & - & & \\
\hline c. $[143 \mathrm{~T}>\mathrm{C}] ;[782 \mathrm{G}>\mathrm{A}]$ & $\begin{array}{l}\text { p. [Leu48Ser]; } \\
\text { [Arg261GIn] }\end{array}$ & $2(0.77)$ & - & - & $\begin{array}{l}2(2 / \\
0)\end{array}$ & 2 (cPKU) & 0 & $22 \mathrm{R} ; 5 \mathrm{NR}$ & $R(39)$ & $\begin{array}{l}R^{a} \\
(38,5)\end{array}$ & - & & \\
\hline c. [143 T > C]; [1045 T > C] & $\begin{array}{l}\text { p. [Leu48Ser]; } \\
\text { [Ser349Pro] }\end{array}$ & $1(0.38)$ & - & - & $\begin{array}{l}1(0 / \\
1)\end{array}$ & 0 & 1 (cPKU) & & R (39) & NR (1) & - & & \\
\hline c. [143 T> C]; [1055delG] & $\begin{array}{l}\text { p. [Leu48Ser]; } \\
\text { [Gly352Valfs*48] }\end{array}$ & $1(0.38)$ & - & - & $\begin{array}{l}\text { 1) }(0 / \\
1)\end{array}$ & 0 & 1 (cPKU) & & R (39) & unk & - & & NR \\
\hline $\begin{array}{l}\text { C. }[143 \mathrm{~T}>\mathrm{C}] ;[1066-11 \mathrm{G}> \\
\text { A] }\end{array}$ & p. [Leu48Ser]; [?] & $3(1.15)$ & - & $\begin{array}{l}\text { 1) }(0 / \\
1)\end{array}$ & $\begin{array}{l}\text { 2) }(0 / \\
2)\end{array}$ & 0 & 3 (1 mPKU, 2 cPKU) & & $\mathrm{R}(39)$ & NR (0) & - & & \\
\hline
\end{tabular}


Table 1 Genotypes observed in French patients with phenylketonuria. Association with clinical phenotypes and response to BH4 loading-test (Continued)

\begin{tabular}{|c|c|c|c|c|c|c|c|c|c|c|c|}
\hline c. $[143 \mathrm{~T}>\mathrm{C}] ;[1162 \mathrm{G}>\mathrm{A}]$ & $\begin{array}{l}\text { p. [Leu48Ser]; } \\
\text { [Val388Met] }\end{array}$ & $1(0.38)$ & - & $\begin{array}{l}1(1 / \\
0)\end{array}$ & - & 1 (mPKU) & 0 & $2 \mathrm{mPKU} R$ & $\mathrm{R}(39)$ & $\begin{array}{l}\text { R } \\
(27,5)\end{array}$ & - \\
\hline $\begin{array}{l}\text { c. [143 T> C]; [442-?_509 } \\
+ \text { ?del] }\end{array}$ & p. [Leu48Ser]; [Ex5del] & $1(0.38)$ & - & - & $\begin{array}{l}\text { 1) }(0 / \\
1)\end{array}$ & 0 & 1 (cPKU) & & $\mathrm{R}(39)$ & unk & - \\
\hline c. [155delT]; ; [781C > T] & $\begin{array}{l}\text { p. [Leu52Cysfs*9]; } \\
{[\text { Arg261*] }}\end{array}$ & $1(0.38)$ & - & - & $\begin{array}{l}\text { 1) }(0 / \\
1)\end{array}$ & 0 & 1 (cPKU) & & unk & NR (1) & - \\
\hline c. $[158 \mathrm{G}>\mathrm{A}] ;$ [?] & p. [Arg53His]; [?] & $1(0.38)$ & - & $\begin{array}{l}1(1 / \\
0)\end{array}$ & - & 1 (mPKU) & 0 & & $\begin{array}{l}\text { unk } \\
(79)\end{array}$ & - & - \\
\hline $\begin{array}{l}\text { c. }[164 \mathrm{~T}>\mathrm{C}] \text {; } ; 1066- \\
11 \mathrm{G}>\mathrm{A}]\end{array}$ & p. [Phe55Ser]; [?] & $1(0.38)$ & - & - & $\begin{array}{l}\text { 1) }(0 / \\
\text { 1) }\end{array}$ & 0 & 1 (cPKU) & & unk & NR (0) & - \\
\hline c. [165delT]; [194 T> C] & $\begin{array}{l}\text { p. [Phe55Leuf**6]; } \\
\text { [lle65Thr] }\end{array}$ & $1(0.38)$ & - & - & $\begin{array}{l}\text { 1) }(0 / \\
1)\end{array}$ & 0 & $1(\mathrm{cPKU})$ & & unk & $\begin{array}{l}\mathrm{R} \\
(25,3)\end{array}$ & - \\
\hline c. [165delT]; $[441+5 G>T]$ & p. [Phe55Leufs*6]; [?] & $2(0.77)$ & - & - & $\begin{array}{l}2(0 / \\
2)\end{array}$ & 0 & $2(2 \mathrm{CPKU})$ & & unk & $N R^{b}$ & - \\
\hline c. [165delT]; $[727 C>T]$ & $\begin{array}{l}\text { p. [Phe55Leufs*6]; } \\
\text { [Arg243*] }\end{array}$ & $1(0.38)$ & - & - & $\begin{array}{l}\text { 1)/ } \\
1)\end{array}$ & 0 & 1 (cPKU) & & unk & NR (1) & - \\
\hline $\begin{array}{l}\text { C. }[165 \mathrm{de} \mid \mathrm{T}] ;[1066-11 \mathrm{G}> \\
\text { A] }\end{array}$ & p. [Phe55Leufs*6]; [?] & $1(0.38)$ & - & - & $\begin{array}{l}\text { 1) }(0 / \\
1)\end{array}$ & 0 & $1(\mathrm{cPKU})$ & & unk & NR (0) & - \\
\hline $\begin{array}{l}\text { C. }[165 \mathrm{del} T] ;[1315+1 \mathrm{G}> \\
\text { A] }\end{array}$ & p. [Phe55Leufs*6]; [?] & $1(0.38)$ & - & - & $\begin{array}{l}1(0 / \\
1)\end{array}$ & 0 & 1 (cPKU) & & unk & NR (0) & - \\
\hline $\begin{array}{l}\text { c. }[168+5 G>C] ;[168+ \\
5 G>C]\end{array}$ & p. [?]; [?] & $1(0.38)$ & - & - & $\begin{array}{l}\text { 1)/ } \\
\text { 1) }\end{array}$ & 0 & $1(\mathrm{cPKU})$ & & unk & unk & - \\
\hline $\begin{array}{l}\text { c. }[168+5 G>C] ;[782 G> \\
\text { A] }\end{array}$ & p. [?]; [Arg261GIn] & $2(0.77)$ & - & - & $\begin{array}{l}2(1 / \\
1)\end{array}$ & 1 (CPKU) minus $61 \%$ & 1 (cPKU) & $\begin{array}{l}3 \text { PKU R; } \\
1 \text { PKU NR }\end{array}$ & unk & $\begin{array}{l}\mathrm{R}^{\mathrm{a}} \\
(38.5)\end{array}$ & - \\
\hline $\begin{array}{l}\text { c. }[168+5 G>C] ;[1-?, 60 \\
+? \text { del }]^{\#}\end{array}$ & p. [?]; [Ex1del] & $1(0.38)$ & - & - & $\begin{array}{l}\text { 1) }(0 / \\
1)\end{array}$ & 0 & 1 (cPKU) & & unk & unk & - \\
\hline c. $[169 G>T]^{\#} ;[194 \mathrm{~T}>C]$ & p. [Glu57*]; [lle65Thr] & $1(0.38)$ & - & - & $\begin{array}{l}\text { 1) }(0 / \\
\text { 1) }\end{array}$ & 0 & 1 (cPKU) & & unk & $\begin{array}{l}\mathrm{R} \\
(25,3)\end{array}$ & $\mathrm{NF}$ \\
\hline c. $[194 \mathrm{~T}>\mathrm{C}] ;[204 \mathrm{~A}>\mathrm{T}]$ & p. [lle65Thr]; [Arg68Ser] & $1(0.38)$ & - & $\begin{array}{l}1(1 / \\
0)\end{array}$ & - & 1 (mPKU) & 0 & $\begin{array}{l}2 \text { PKU R; } \\
1 \text { PKU NR }\end{array}$ & $\begin{array}{l}\mathrm{R} \\
(25.3)\end{array}$ & $\mathrm{R}(87)$ & - \\
\hline c. $[194 \mathrm{~T}>\mathrm{C}] ;[331 \mathrm{C}>\mathrm{T}]$ & p. [lle65Thr]; [Arg111*] & $1(0.38)$ & - & - & $\begin{array}{l}1(0 / \\
1)\end{array}$ & 0 & $1(\mathrm{cPKU})$ & & $\begin{array}{l}\mathrm{R} \\
(25.3)\end{array}$ & NR (1) & - \\
\hline c. $[194 \mathrm{~T}>\mathrm{C}] ;[386 \mathrm{~A}>\mathrm{G}]$ & p. [lle65Thr]; [Asp129Gly] & $1(0.38)$ & - & - & $\begin{array}{l}1(0 / \\
1)\end{array}$ & 0 & 1 (cPKU) & & $\begin{array}{l}\mathrm{R} \\
(25.3)\end{array}$ & $\mathrm{R}$ & - \\
\hline c. $[194 \mathrm{~T}>\mathrm{C}] ;[473 \mathrm{G}>\mathrm{A}]$ & p. [lle65Thr]; [Arg158GIn] & $1(0.38)$ & - & - & $\begin{array}{l}1(0 / \\
1)\end{array}$ & 0 & 1 (cPKU) & & $\begin{array}{l}\mathrm{R} \\
(25.3)\end{array}$ & $R(10)$ & - \\
\hline c. $[194 \mathrm{~T}>\mathrm{C}] ;[526 \mathrm{C}>\mathrm{T}]$ & p. [lle65Thr]; [Arg176*] & $1(0.38)$ & - & - & $\begin{array}{l}1(0 / \\
1)\end{array}$ & 0 & 1 (cPKU) & & $\begin{array}{l}\mathrm{R} \\
(25.3)\end{array}$ & NR (1) & - \\
\hline c. $[194 \mathrm{~T}>\mathrm{C}] ;[782 \mathrm{G}>\mathrm{A}]$ & p. [lle65Thr]; [Arg261GIn] & $3(1.15)$ & - & - & & 0 & $3(3 \mathrm{cPKU})$ & & & & - \\
\hline
\end{tabular}


Table 1 Genotypes observed in French patients with phenylketonuria. Association with clinical phenotypes and response to BH4 loading-test (Continued)

\begin{tabular}{|c|c|c|c|c|c|c|c|c|c|c|c|c|}
\hline & & & & & $\begin{array}{l}3(0 / \\
3)\end{array}$ & & & & $\begin{array}{l}\mathrm{R} \\
(25.3)\end{array}$ & $\begin{array}{l}\mathrm{R}^{\mathrm{a}} \\
(38,5)\end{array}$ & & \\
\hline c. [194 T>C]; [782G > C] & p. [lle65Thr]; [Arg261Pro] & $1(0.38)$ & - & - & $\begin{array}{l}1(0 / \\
1)\end{array}$ & 0 & 1 (cPKU) & & $\begin{array}{l}\mathrm{R} \\
(25.3)\end{array}$ & unk & - & \\
\hline c. [194 T > C]; [829 T > G] & p. [lle65Thr]; [Tyr277Asp] & $1(0.38)$ & - & $\begin{array}{l}\text { 1) }(0 / \\
1)\end{array}$ & - & 0 & 1 (mPKU) & & $\begin{array}{l}\mathrm{R} \\
(25.3)\end{array}$ & $\begin{array}{l}\text { unk } \\
(0-12)\end{array}$ & - & \\
\hline c. [194 T > C]; [838G > A] & p. [lle65Thr]; [Glu280Lys] & $1(0.38)$ & - & - & $\begin{array}{l}1(0 / \\
1)\end{array}$ & 0 & 1 (cPKU) & & $\begin{array}{l}R \\
(25.3)\end{array}$ & $\begin{array}{l}\text { NR } \\
(1.95)\end{array}$ & - & \\
\hline c. $[194 \mathrm{~T}>\mathrm{C}] ;[842 \mathrm{C}>\mathrm{T}]$ & p. [lle65Thr]; [Pro281Leu] & $2(0.77)$ & - & - & $\begin{array}{l}2(0 / \\
2)\end{array}$ & 0 & 2 (2 cPKU) & & $\begin{array}{l}\mathrm{R} \\
(25.3)\end{array}$ & NR (1) & - & \\
\hline c. $[194 \mathrm{~T}>\mathrm{C}] ;[842+1 \mathrm{G}>\mathrm{A}]$ & p. [lle65Thr]; [?] & $1(0.38)$ & - & - & $\begin{array}{l}1(0 / \\
1)\end{array}$ & 0 & 1 (cPKU) & & $\begin{array}{l}\mathrm{R} \\
(25.3)\end{array}$ & unk & - & \\
\hline c. $[194 \mathrm{~T}>\mathrm{C}] ;[844 \mathrm{G}>\mathrm{A}]$ & $\begin{array}{l}\text { p. [lle65Thr]; } \\
\text { [Asp282Asn] }\end{array}$ & $1(0.38)$ & - & - & $\begin{array}{l}\text { 1)/ } \\
\text { 1) }\end{array}$ & 0 & 1 (cPKU) & & $\begin{array}{l}\mathrm{R} \\
(25.3)\end{array}$ & $\begin{array}{l}\text { unk } \\
(2)\end{array}$ & - & \\
\hline c. [194 T > C]; [898G > T] & p. [lle65Thr]; [Ala300Ser] & $1(0.38)$ & $\begin{array}{l}1(1 / \\
0)\end{array}$ & - & - & $1(\mathrm{mHP})$ & 0 & $2 \mathrm{mHPR}$ & $\begin{array}{l}\mathrm{R} \\
(25.3)\end{array}$ & $\mathrm{R}(31)$ & - & \\
\hline c. [194 T > C]; $[912$ + 1G > A] & p. [lle65Thr]; [?] & $1(0.38)$ & - & - & $\begin{array}{l}1(0 / \\
1)\end{array}$ & 0 & 1 (cPKU) & & $\begin{array}{l}\mathrm{R} \\
(25.3)\end{array}$ & unk & - & \\
\hline c. [194 T > C]; [916A > G] & p. [lle65Thr]; [l306Val] & $1(0.38)$ & - & $\begin{array}{l}1(1 / \\
0)\end{array}$ & - & 1 (mPKU) & 0 & $2 \mathrm{R}$ & $\begin{array}{l}R \\
(25.3)\end{array}$ & $\begin{array}{l}\text { unk } \\
(12- \\
39)\end{array}$ & - & \\
\hline c. [194 T > C]; [932 T > C] & p. [lle65Thr]; [Leu311Pro] & $1(0.38)$ & - & - & $\begin{array}{l}1(0 / \\
1)\end{array}$ & 0 & 1 (cPKU) & & $\begin{array}{l}R \\
(25.3)\end{array}$ & NR (1) & - & \\
\hline c. $[194 \mathrm{~T}>\mathrm{C}] ;[1042 \mathrm{C}>\mathrm{G}]$ & p. [lle65Thr]; [Leu348Val] & $1(0.38)$ & - & $\begin{array}{l}1(1 / \\
0)\end{array}$ & - & 1 (mPKU) & 0 & $\begin{array}{l}5 \text { PKU R; } \\
1 \text { PKU NR }\end{array}$ & $\begin{array}{l}\mathrm{R} \\
(25.3)\end{array}$ & $\mathrm{R}(41)$ & - & \\
\hline c. [194 T>C]; [1045 T>C] & p. [lle65Thr]; [Ser349Pro] & $1(0.38)$ & - & - & $\begin{array}{l}1(0 / \\
1)\end{array}$ & 0 & 1 (cPKU) & & $\begin{array}{l}\mathrm{R} \\
(25.3)\end{array}$ & NR (1) & - & \\
\hline $\begin{array}{l}\text { c. }[194 \mathrm{~T}>\mathrm{C}] ;[1066-11 \mathrm{G}> \\
\mathrm{A}]\end{array}$ & p. [lle65Thr]; [?] & $4(1.53)$ & - & - & $\begin{array}{l}4(0 / \\
4)\end{array}$ & 0 & 4 (4 cPKU) & & $\begin{array}{l}\mathrm{R} \\
(25.3)\end{array}$ & NR (0) & - & \\
\hline c. [194 T>C]; [1169A > G] & p. [lle65Thr]; [Glu390Gly] & $2(0.77)$ & - & $\begin{array}{l}1(1 / \\
0)\end{array}$ & $\begin{array}{l}1(1 / \\
0)\end{array}$ & $\begin{array}{l}2 \text { (mPKU, } \\
\text { cPKU) }\end{array}$ & 0 & 3 PKU R & $\begin{array}{l}\mathrm{R} \\
(25.3)\end{array}$ & $\begin{array}{l}\mathrm{R} \\
(72.5)\end{array}$ & - & \\
\hline $\begin{array}{l}\text { c. }[194 \mathrm{~T}>\mathrm{C}] ;[1169 \mathrm{~A}>\mathrm{G}] ; \\
{[1171 \mathrm{~A}>\mathrm{G}]^{*}}\end{array}$ & $\begin{array}{l}\text { p. [lle65Thr]; ([Glu390Gly)]; } \\
\text { [(Ser391Gly)] }\end{array}$ & $1(0.38)$ & - & - & $\begin{array}{l}1(1 / \\
0)\end{array}$ & 1 (cPKU) & 0 & & $\begin{array}{l}\mathrm{R} \\
(25.3)\end{array}$ & $\begin{array}{l}R \\
(72.5)\end{array}$ & unk & \\
\hline c. $[194 \mathrm{~T}>\mathrm{C}] ;[1208 \mathrm{C}>\mathrm{T}]$ & p. [lle65Thr]; [Ala403Val] & $4(1.53)$ & $\begin{array}{l}2(2 / \\
0)\end{array}$ & $\begin{array}{l}1(0 / \\
1)\end{array}$ & - & $2(2 \mathrm{mHP})$ & $\begin{array}{l}1 \text { (PKUa) minus } \\
15 \%\end{array}$ & $1 \mathrm{mHP} R$ & $\begin{array}{l}\mathrm{R} \\
(25.3)\end{array}$ & $\mathrm{R}(32)$ & - & \\
\hline c. $[194 \mathrm{~T}>\mathrm{C}] ;[1222 \mathrm{C}>\mathrm{T}]$ & p. [lle65Thr]; [Arg408Trp] & $2(0.77)$ & - & - & $\begin{array}{l}2(1 / \\
1)\end{array}$ & 1 (cPKU) minus $32 \%$ & $\begin{array}{l}1(\mathrm{cPKU}) \text { minus } \\
14 \%\end{array}$ & $9 \mathrm{R} ; 8 \mathrm{NR}$ & $\begin{array}{l}\mathrm{R} \\
(25.3)\end{array}$ & NR (1) & - & \\
\hline $\begin{array}{l}\text { c. }\left[196 \mathrm{G}>T^{\#} ;[1315+\right. \\
\text { 1G }>A]\end{array}$ & p. [Glu66*]; [?] & $1(0.38)$ & - & - & $\begin{array}{l}\text { 1) }(0 / \\
1)\end{array}$ & 0 & $1(\mathrm{cPKU})$ & & unk & NR (0) & - & NR \\
\hline
\end{tabular}


Table 1 Genotypes observed in French patients with phenylketonuria. Association with clinical phenotypes and response to BH4 loading-test (Continued)

\begin{tabular}{|c|c|c|c|c|c|c|c|c|c|c|c|c|c|}
\hline c. $[204 \mathrm{~A}>\mathrm{T}] ;[204 \mathrm{~A}>\mathrm{T}]$ & p. [Arg68Ser]; [Arg68Ser] & $1(0.38)$ & - & $\begin{array}{l}1(1 / \\
0)\end{array}$ & - & 1 (mPKU) & 0 & $1 \mathrm{mPKU} \mathrm{R}$ & $\mathrm{R}(87)$ & $\mathrm{R}(87)$ & - & & \\
\hline $\begin{array}{l}\text { c. }[204 \mathrm{~A}>\mathrm{T}] ; \\
{\left[398 \_401 \text { delATCA] }\right.}\end{array}$ & $\begin{array}{l}\text { p. [Arg68Ser]; } \\
\text { [Asn133Argfs*61] }\end{array}$ & $1(0.38)$ & - & - & $\begin{array}{l}1(0 / \\
1)\end{array}$ & 0 & 1 (cPKU) & & $\mathrm{R}(87)$ & unk & - & & \\
\hline $\begin{array}{l}\text { c. }[204 \mathrm{~A}>\mathrm{T}] ;[526 \mathrm{C}>\mathrm{T}] \\
{[707-7 \mathrm{~A}>\mathrm{T}]}\end{array}$ & $\begin{array}{l}\text { p. }[\operatorname{Arg} 685 e r] ; \\
{\left[\left(\operatorname{Arg} 176^{*}\right)\right] ;[(?)]}\end{array}$ & $1(0.38)$ & - & $\begin{array}{l}1(1 / \\
0)\end{array}$ & - & $1(\mathrm{mPKU})$ & 0 & no data & $\mathrm{R}(87)$ & NR (1) & unk & & \\
\hline c. $[204 \mathrm{~A}>\mathrm{T}] ;[611 \mathrm{~A}>\mathrm{G}]$ & $\begin{array}{l}\text { p. [Arg68Ser]; } \\
\text { [Tyr204Cys] }\end{array}$ & $1(0.38)$ & - & $\begin{array}{l}1(0 / \\
1)\end{array}$ & - & 0 & 1 (mPKU) & & $\mathrm{R}(87)$ & unk & - & & \\
\hline c. $[204 \mathrm{~A}>\mathrm{T}] ;[814 \mathrm{G}>\mathrm{T}]$ & p. [Arg68Ser]; [Gly272*] & $1(0.38)$ & - & $\begin{array}{l}1(1 / \\
0)\end{array}$ & - & 1 (mPKU) & 0 & 1CPKU R & $\mathrm{R}(87)$ & NR (1) & - & & \\
\hline c. $[204 \mathrm{~A}>\mathrm{T}] ;$ [1045 T>C] & $\begin{array}{l}\text { p. [Arg68Ser]; } \\
\text { [Ser349Pro] }\end{array}$ & $1(0.38)$ & $\begin{array}{l}1(1 / \\
0)\end{array}$ & - & - & 1 (mHP) & 0 & no data & $\mathrm{R}(87)$ & NR (1) & - & & \\
\hline c. [204A > T]; [1055delG] & $\begin{array}{l}\text { p. [Arg68Ser]; } \\
\text { [Gly352Valfs*48] }\end{array}$ & $1(0.38)$ & - & $\begin{array}{l}1(0 / \\
1)\end{array}$ & - & 0 & 1 (mPKU) & & $\mathrm{R}(87)$ & unk & - & & NR \\
\hline c. $[204 \mathrm{~A}>\mathrm{T}] ;[1162 \mathrm{G}>\mathrm{A}]$ & $\begin{array}{l}\text { p. [Arg68Ser]; } \\
\text { Val388Met] }\end{array}$ & $1(0.38)$ & $\begin{array}{l}1(1 / \\
0)\end{array}$ & - & - & 1 (mHP) & 0 & no data & R (87) & $\begin{array}{l}\mathrm{R} \\
(27.5)\end{array}$ & - & & \\
\hline c. [204A > T]; [1222C > T] & $\begin{array}{l}\text { p. [Arg68Ser]; } \\
\text { [Arg408Trp] }\end{array}$ & $2(0.77)$ & - & $\begin{array}{l}1(0 / \\
1)\end{array}$ & $\begin{array}{l}1(0 / \\
1)\end{array}$ & 0 & $2(\mathrm{mPKU}, \mathrm{cPKU})$ & & $\mathrm{R}(87)$ & NR (1) & - & & \\
\hline $\begin{array}{l}\text { c. }[204 \mathrm{~A}>\mathrm{T}] ;[1315+1 \mathrm{G}> \\
\text { A] }\end{array}$ & p. [Arg68Ser]; [?] & $1(0.38)$ & - & $\begin{array}{l}1(0 / \\
1)\end{array}$ & - & 0 & 1 (mPKU) & & $\mathrm{R}(87)$ & $N R(0)$ & - & & \\
\hline $\begin{array}{l}\text { c. }[261 C>A] ;[1065+1 G> \\
\text { A] }\end{array}$ & p. [Ser87Arg]; [?] & $1(0.38)$ & $\begin{array}{l}1(0 / \\
1)\end{array}$ & - & - & 0 & $\begin{array}{l}1 \text { (mHP, HO } 2.4 \text { mg } \\
\% \text {, minus } 8 \% \text { ) }\end{array}$ & & $\mathrm{R}(82)$ & unk & - & & \\
\hline $\begin{array}{l}\text { c. }\left[266 \_267 \mathrm{ins} C\right] ;[842 \mathrm{C}> \\
\text { T] }\end{array}$ & $\begin{array}{l}\text { p. [Ala90Cysfs*12]; } \\
\text { [Pro281Leu] }\end{array}$ & $1(0.38)$ & - & - & $\begin{array}{l}1(1 / \\
0)\end{array}$ & 1 (cPKU) minus $36 \%$ & 0 & no data & unk & $N R(1)$ & - & unclear & \\
\hline $\begin{array}{l}\text { c. }\left[284 \_286 \text { delTCA]; }[441+\right. \\
5 G>T]\end{array}$ & p. [lle95del]; [?] & $1(0.38)$ & - & - & $\begin{array}{l}1(0 / \\
1)\end{array}$ & 0 & 1 (cPKU) & & unk & $N R^{b}$ & - & & \\
\hline $\begin{array}{l}\text { c. }\left[284 \_286 d e l T C A\right] ; \\
{[1243 G>A]}\end{array}$ & p. [lle95del]; [Asp415Asn] & $1(0.38)$ & $\begin{array}{l}1(1 / \\
0)\end{array}$ & - & - & 1 (mHP) & 0 & no data & unk & $\mathrm{R}(93)$ & - & & \\
\hline c. $[311 \mathrm{C}>\mathrm{A}] ;[442-5 \mathrm{C}>\mathrm{G}]$ & p. [Ala104Asp]; [?] & $1(0.38)$ & - & $\begin{array}{l}1(0 / \\
1)\end{array}$ & - & 0 & 1 (mPKU) & & R (26) & R & - & & \\
\hline c. $[311 \mathrm{C}>\mathrm{A}] ;[1222 \mathrm{C}>\mathrm{T}]$ & $\begin{array}{l}\text { p. [Ala104Asp]; } \\
\text { [Arg408Trp] }\end{array}$ & $1(0.38)$ & - & - & $\begin{array}{l}1(0 / \\
1)\end{array}$ & 0 & 1 (cPKU) & & $\mathrm{R}(26)$ & NR (1) & - & & \\
\hline c. $[331 \mathrm{C}>\mathrm{T}] ;[782 \mathrm{G}>\mathrm{A}]$ & p. [Arg111*]; [Arg261GIn] & $1(0.38)$ & - & - & $\begin{array}{l}1(0 / \\
1)\end{array}$ & 0 & $1(\mathrm{CPKU})$ & & NR (1) & $\begin{array}{l}R^{a} \\
(38.5)\end{array}$ & - & & \\
\hline c. $[331 C>T] ;[1222 C>T]$ & p. [Arg111*]; [Arg408Trp] & $1(0.38)$ & - & - & $\begin{array}{l}1(0 / \\
1)\end{array}$ & 0 & 1 (cPKU) & & NR (1) & $N R(1)$ & - & & \\
\hline \multirow[t]{2}{*}{ c. $[434 \mathrm{~A}>\mathrm{T}] ;[1222 \mathrm{C}>\mathrm{T}]$} & $\begin{array}{l}\text { p. [Asp145Val]; } \\
\text { [Arg408Trp] }\end{array}$ & $1(0.38)$ & $\begin{array}{l}1(1 / \\
0)\end{array}$ & - & - & 1 (mHP) & 0 & no data & unk & NR (1) & - & \multirow{2}{*}{\multicolumn{2}{|c|}{ R }} \\
\hline & p. [?]; [?] & $1(0.38)$ & - & - & & 0 & 1 (cPKU) & & $N R^{b}$ & $N R^{b}$ & - & & \\
\hline
\end{tabular}


Table 1 Genotypes observed in French patients with phenylketonuria. Association with clinical phenotypes and response to BH4 loading-test (Continued)

\begin{tabular}{|c|c|c|c|c|c|c|c|c|c|c|c|c|}
\hline $\begin{array}{l}\text { c. }[441+5 G>T] ;[441+ \\
5 G>T]\end{array}$ & & & & & $\begin{array}{l}\text { 1) }(0 / \\
1)\end{array}$ & & & & & & & \\
\hline $\begin{array}{l}\text { C. }[441+5 G>T] ;[473 G> \\
\text { A] }\end{array}$ & p. [?]; [Arg158GIn] & $2(0.77)$ & - & - & $\begin{array}{l}2(0 / \\
2)\end{array}$ & 0 & $2(2 \mathrm{CPKU})$ & & $N R^{b}$ & $R(10)$ & - & \\
\hline $\begin{array}{l}\text { C. }[441+5 G>T] ;[631 C> \\
\text { A] }\end{array}$ & p. [?]; [Pro211Thr] & $1(0.38)$ & $\begin{array}{l}1(1 / \\
0)\end{array}$ & - & - & $1(\mathrm{mHP})$ & 0 & no data & $N R^{b}$ & $\mathrm{R}(72)$ & - & \\
\hline $\begin{array}{l}\text { C. }[441+5 G>T] ;[782 G> \\
\text { A] }\end{array}$ & p. [?]; [Arg261Gln] & $2(0.77)$ & - & - & $\begin{array}{l}2(0 / \\
2)\end{array}$ & 0 & $2(2 \mathrm{cPKU})$ & & $N R^{b}$ & $\begin{array}{l}\mathrm{R}^{\mathrm{a}} \\
(38.5)\end{array}$ & - & \\
\hline $\begin{array}{l}\text { c. }[441+5 G>T] ;[838 G> \\
\text { A] }\end{array}$ & p. [?]; [Glu280Lys] & $1(0.38)$ & - & - & $\begin{array}{l}1(0 / \\
1)\end{array}$ & 0 & $1(\mathrm{cPKU})$ & & $N R_{b}$ & $\begin{array}{l}\mathrm{NR} \\
(1.95)\end{array}$ & - & \\
\hline $\begin{array}{l}\text { c. }[441+5 G>T] ;[1045 \mathrm{~T}> \\
\mathrm{G}]\end{array}$ & p. [?]; [Ser349Ala] & $1(0.38)$ & - & - & $\begin{array}{l}1(1 / \\
0)\end{array}$ & 1 (cPKU) minus $41 \%$ & 0 & no data & $N R_{b}$ & unk & - & $\mathrm{R}$ \\
\hline $\begin{array}{l}\text { C. }[442-2 A>C]^{\# ;} ;[442-2 A> \\
\text { C] }]^{\#}\end{array}$ & p. [?]; [?] & $1(0.38)$ & - & - & $\begin{array}{l}1(0 / \\
1)\end{array}$ & 0 & 1 (cPKU) & & unk & unk & - & \\
\hline c. $[464 G>C] ;[1223 G>A]$ & $\begin{array}{l}\text { p. [Arg155Pro]; } \\
\text { [Arg408GIn] }\end{array}$ & $1(0.38)$ & - & - & $\begin{array}{l}1(0 / \\
1)\end{array}$ & 0 & 1 (cPKU) & & unk & $\begin{array}{l}\mathrm{R} \\
(49.7)\end{array}$ & - & \\
\hline $\begin{array}{l}\text { c. [464G > C]; [510-?-706 } \\
\text { + ?del] }\end{array}$ & p. [Arg155Pro]; [Ex6del] & $1(0.38)$ & - & - & $\begin{array}{l}1(0 / \\
1)\end{array}$ & 0 & 1 (cPKU) & & unk & unk & - & \\
\hline c. $[472 C>T] ;[472 C>T]$ & $\begin{array}{l}\text { p. [Arg158Trp]; } \\
\text { [Arg158Trp] }\end{array}$ & $1(0.38)$ & - & $\begin{array}{l}\text { 1 }(0 / \\
\text { 1) }\end{array}$ & - & 0 & $1(\mathrm{mPKU})$ & & unk & unk & - & \\
\hline c. $[472 C>T] ;[754 C>T]$ & $\begin{array}{l}\text { p. [Arg158Trp]; } \\
\text { [Arg252Trp] }\end{array}$ & $1(0.38)$ & - & - & $\begin{array}{l}\text { 1)/ } \\
\text { 1) }\end{array}$ & 0 & 1 (cPKU) & & unk & NR (1) & - & \\
\hline c. $[473 \mathrm{G}>\mathrm{A}] ;[473 \mathrm{G}>\mathrm{A}]$ & $\begin{array}{l}\text { p. [Arg158GIn]; } \\
\text { [Arg158GIn] }\end{array}$ & $1(0.38)$ & - & - & $\begin{array}{l}\text { 1) }(0 / \\
1)\end{array}$ & 0 & 1 (cPKU) & & $R(10)$ & $R(10)$ & - & \\
\hline c. $[473 \mathrm{G}>\mathrm{A}] ;[526 \mathrm{C}>\mathrm{T}]$ & p. [Arg158GIn]; [Arg176*] & $1(0.38)$ & - & - & $\begin{array}{l}1(0 / \\
1)\end{array}$ & 0 & 1 (cPKU) & & $\mathrm{R}(10)$ & NR (1) & - & \\
\hline c. $[473 \mathrm{G}>\mathrm{A}] ;[\mathrm{814G}>\mathrm{T}]$ & p. [Arg158GIn]; [Gly272*] & $1(0.38)$ & - & - & $\begin{array}{l}\text { 1)/ } \\
\text { 1) }\end{array}$ & 0 & 1 (cPKU) & & $R(10)$ & NR (1) & - & \\
\hline $\begin{array}{l}\text { C. }[473 \mathrm{G}>\mathrm{A}] ;[1066-11 \mathrm{G}> \\
\mathrm{A}]\end{array}$ & p. [Arg158GIn]; [?] & $3(1.15)$ & - & - & $\begin{array}{l}3(0 / \\
3)\end{array}$ & 0 & 3 (3 cPKU) & & $R(10)$ & NR (0) & - & \\
\hline c. $[473 G>A] ;[1169 A>G]$ & $\begin{array}{l}\text { p. [Arg158Gln]; } \\
\text { [Glu390Gly] }\end{array}$ & $1(0.38)$ & - & $\begin{array}{l}1(1 / \\
0)\end{array}$ & - & $1(\mathrm{mPKU})$ & 0 & $5 R$ & $\mathrm{R}(10)$ & $\begin{array}{l}R \\
(72.5)\end{array}$ & - & \\
\hline c. $[473 G>A] ;[1222 C>T]$ & $\begin{array}{l}\text { p. [Arg158Gln]; } \\
\text { [Arg408Trp] }\end{array}$ & $1(0.38)$ & - & - & $\begin{array}{l}1(0 / \\
1)\end{array}$ & 0 & 1 (cPKU) & & $R(10)$ & NR (1) & - & \\
\hline $\begin{array}{l}\text { c. }[473 G>A] ;[169-? .352+ \\
\text { ?del] }\end{array}$ & p. [Arg158GIn]; [Ex3del] & $1(0.38)$ & - & - & $\begin{array}{l}1(0 / \\
1)\end{array}$ & 0 & 1 (cPKU) & & $R(10)$ & unk & - & \\
\hline c. $[473 \mathrm{G}>\mathrm{T}] ;[782 \mathrm{G}>\mathrm{C}]$ & $\begin{array}{l}\text { p. [Arg158Trp]; } \\
\text { [Arg261Pro] }\end{array}$ & $1(0.38)$ & - & - & $\begin{array}{l}1(0 / \\
1)\end{array}$ & 0 & 1 (cPKU) & & unk & unk & - & \\
\hline c. $[491 \mathrm{~T}>\mathrm{C}] ;[1241 \mathrm{~A}>\mathrm{G}]$ & & $1(0.38)$ & - & & - & 1 (mPKU) & 0 & no data & unk & $R^{a}(36)$ & - & \\
\hline
\end{tabular}


Table 1 Genotypes observed in French patients with phenylketonuria. Association with clinical phenotypes and response to BH4 loading-test (Continued)

\begin{tabular}{|c|c|c|c|c|c|c|c|c|c|c|c|c|}
\hline & $\begin{array}{l}\text { p. [lle164Thr]; } \\
\text { [Tyr414Cys] }\end{array}$ & & & $\begin{array}{l}1(1 / \\
0)\end{array}$ & & & & & & & & \\
\hline c. $[493 G>A] ;[493 G>A]$ & $\begin{array}{l}\text { p. [Ala165Thr]; } \\
\text { [Ala165Thr] }\end{array}$ & $2(0.77)$ & - & - & $\begin{array}{l}2(1 / \\
1)\end{array}$ & 1 (cPKU) minus $36 \%$ & 1 (cPKU) minus $0 \%$ & no data & unk & unk & - & unclear unclear \\
\hline c. $[493 \mathrm{G}>\mathrm{C}] ;[1169 \mathrm{~A}>\mathrm{G}]$ & p. [Ala165Pro]; [Glu390Gly] & $1(0.38)$ & - & $\begin{array}{l}1(1 / \\
0)\end{array}$ & - & 1 (mPKU) & 0 & no data & unk & $\begin{array}{l}R \\
(72.5)\end{array}$ & - & \\
\hline $\begin{array}{l}\text { c. }[500 \mathrm{~A}>\mathrm{T}] ;[1066-11 \mathrm{G}> \\
\text { A] }\end{array}$ & p. [Asn167\|le]; [?] & $1(0.38)$ & - & $\begin{array}{l}1(1 / \\
0)\end{array}$ & - & 1 (mPKU) & 0 & no data & unk & NR (0) & - & $\mathrm{R}$ \\
\hline c. $[504 C>A]^{\#} ;[898 G>T]$ & p. [Tyr168*]; [Ala300Ser] & $1(0.38)$ & $\begin{array}{l}1(1 / \\
0)\end{array}$ & - & - & $1(\mathrm{mHP})$ & 0 & no data & unk & $R(31)$ & - & NR \\
\hline c. $[506 \mathrm{G}>\mathrm{A}] ;[1066-3 \mathrm{C}>\mathrm{T}]$ & p. [Arg169His]; [?] & $1(0.38)$ & $\begin{array}{l}1(1 / \\
0)\end{array}$ & - & - & $1(\mathrm{mHP})$ & 0 & no data & unk & $\mathrm{R}$ & - & \\
\hline $\begin{array}{l}\text { c. }[509+1 G>A] ;[561 G> \\
\text { A] }\end{array}$ & p. [?]; [Trp187*] & $1(0.38)$ & - & - & $\begin{array}{l}\text { 1) }(0 / \\
1)\end{array}$ & 0 & 1 (cPKU) & & unk & NR (0) & - & \\
\hline c. $[529 \mathrm{G}>\mathrm{C}] ;[561 \mathrm{G}>\mathrm{A}]$ & p. [Val177Leu]; [Trp187*] & $1(0.38)$ & - & $\begin{array}{l}1(1 / \\
0)\end{array}$ & - & 1 (mPKU) & 0 & no data & unk & NR (0) & - & $\mathrm{R}$ \\
\hline c. $[569 \mathrm{~T}>\mathrm{C}] ;[\mathrm{796 \textrm {A }}>\mathrm{C}]^{\#}$ & p. [Val190Ala]; [Thr266P] & $1(0.38)$ & - & - & $\begin{array}{l}\text { 1) }(0 / \\
1)\end{array}$ & 0 & 1 (cPKU) & & $\begin{array}{l}R \\
(110)\end{array}$ & unk & - & \\
\hline $\begin{array}{l}\text { c. [580_581delCT]; } \\
{[1042 \mathrm{C}>\mathrm{G}]}\end{array}$ & $\begin{array}{l}\text { p. [Leu194Gluf*5]; } \\
\text { [Leu348Val] }\end{array}$ & $1(0.38)$ & - & - & $\begin{array}{l}1(0 / \\
1)\end{array}$ & 0 & 1 (cPKU) & & unk & $\mathrm{R}(41)$ & - & \\
\hline c. $[581 \mathrm{~T}>\mathrm{C}] ;[842 \mathrm{C}>\mathrm{T}]$ & $\begin{array}{l}\text { p. [Leu194Pro]; } \\
\text { [Pro281Leu] }\end{array}$ & $1(0.38)$ & - & $\begin{array}{l}\text { 1) }(0 / \\
1)\end{array}$ & - & 0 & 1 (mPKU) & & unk & NR (1) & - & \\
\hline $\begin{array}{l}\text { c. [593_614del22]; [969+ } \\
\text { 1G > A] }\end{array}$ & p. [Tyr198Cysfs*136]; [?] & $1(0.38)$ & - & - & $\begin{array}{l}1(0 / \\
1)\end{array}$ & 0 & 1 (cPKU) & & unk & unk & - & \\
\hline c. $[631 \mathrm{C}>\mathrm{A}] ;[782 \mathrm{G}>\mathrm{A}]$ & $\begin{array}{l}\text { p. [Pro211Thr]; } \\
\text { [Arg261Gln] }\end{array}$ & $2(0.77)$ & - & $\begin{array}{l}1(1 / \\
0)\end{array}$ & $\begin{array}{l}1(1 / \\
0)\end{array}$ & 2 (mPKU, cPKU) & 0 & $4 \mathrm{mHPR}$ & $\mathrm{R}(72)$ & $\begin{array}{l}\mathrm{R}^{\mathrm{a}} \\
(38,5)\end{array}$ & - & \\
\hline $\begin{array}{l}\text { C. }[631 \mathrm{C}>\mathrm{A}] ; \text {; }[1066-11 \mathrm{G}> \\
\mathrm{A}]\end{array}$ & p. [Pro211Thr]; [?] & $1(0.38)$ & - & $\begin{array}{l}1(1 / \\
0)\end{array}$ & - & 1 (mPKU) & 0 & $10 R$ & $\mathrm{R}(72)$ & NR (0) & - & \\
\hline $\begin{array}{l}\text { C. [632delC]; [1066-11G > } \\
\text { A] }\end{array}$ & p. [Pro211Hisfs*130]; [?] & $1(0.38)$ & - & - & $\begin{array}{l}1(0 / \\
1)\end{array}$ & 0 & 1 (cPKU) & & unk & NR $(0)$ & - & \\
\hline $\begin{array}{l}\text { c. }[635 \mathrm{~T}>\mathrm{C}] ;[1066-11 \mathrm{G}> \\
\text { A] }\end{array}$ & p. [Leu212Pro]; [?] & $1(0.38)$ & - & - & $\begin{array}{l}1(0 / \\
1)\end{array}$ & 0 & 1 (cPKU) & & unk & NR $(0)$ & - & \\
\hline c. $[638 \mathrm{~T}>\mathrm{C}] ;[638 \mathrm{~T}>\mathrm{C}]$ & $\begin{array}{l}\text { p. [Leu213Pro]; } \\
\text { [Leu213Pro] }\end{array}$ & $1(0.38)$ & - & - & $\begin{array}{l}1(0 / \\
1)\end{array}$ & 0 & $1(\mathrm{cPKU})$ & & unk & unk & - & \\
\hline c. $[638 \mathrm{~T}>\mathrm{C}] ;[\mathrm{727C}>\mathrm{T}]$ & p. [Leu213Pro]; [Arg243*] & $1(0.38)$ & - & - & $\begin{array}{l}1(0 / \\
1)\end{array}$ & 0 & 1 (cPKU) & & unk & NR (1) & - & \\
\hline c. $[648 \mathrm{C}>\mathrm{G}] ;[838 \mathrm{G}>\mathrm{A}]$ & p. [Tyr216*]; [Glu280Lys] & $1(0.38)$ & - & - & $\begin{array}{l}1(0 / \\
1)\end{array}$ & 0 & 1 (cPKU) & & unk & $\begin{array}{l}\text { NR } \\
(1.95)\end{array}$ & - & NR \\
\hline c. $[653 \mathrm{G}>$ T]; $[809 \mathrm{G}>\mathrm{A}]$ & & $1(0.38)$ & - & - & & 0 & 1 (cPKU) & & unk & & - & \\
\hline
\end{tabular}


Table 1 Genotypes observed in French patients with phenylketonuria. Association with clinical phenotypes and response to BH4 loading-test (Continued)

\begin{tabular}{|c|c|c|c|c|c|c|c|c|c|c|c|c|}
\hline & $\begin{array}{l}\text { p. [Gly218Val]; } \\
\text { [Arg270Lys] }\end{array}$ & & & & $\begin{array}{l}\text { 1) }(0 / \\
1)\end{array}$ & & & & & $\begin{array}{l}\text { NR } \\
(2.1)\end{array}$ & & \\
\hline $\begin{array}{l}\text { c. }[653 G>T] ;[1066-11 G> \\
\text { A] }\end{array}$ & p. [Gly218Val]; [?] & $1(0.38)$ & - & - & $\begin{array}{l}\text { 1) }(0 / \\
1)\end{array}$ & 0 & 1 (cPKU) & & unk & $\mathrm{NR}(0)$ & - & \\
\hline $\begin{array}{l}\text { c. [663_664delAG]; [912+ } \\
1 \mathrm{G}>\mathrm{A}]\end{array}$ & p. [Asp222*]; [?] & $1(0.38)$ & - & - & $\begin{array}{l}1(0 / \\
1)\end{array}$ & 0 & 1 (cPKU) & & NR (1) & unk & - & \\
\hline c. $[671 \mathrm{~T}>\mathrm{C}] ;[671 \mathrm{~T}>\mathrm{C}]$ & p. [lle224Thr]; [lle224Thr] & $2(0.77)$ & - & - & $\begin{array}{l}2(0 / \\
2)\end{array}$ & 0 & $2(2 \mathrm{cPKU})$ & & unk & unk & - & \\
\hline $\begin{array}{l}\text { c. }[682 \mathrm{G}>\mathrm{A}]^{\#} ;[1066- \\
11 \mathrm{G}>\mathrm{A}]\end{array}$ & p. [Glu228Lys]; [?] & $1(0.38)$ & - & - & $\begin{array}{l}\text { 1) }(0 / \\
1)\end{array}$ & 0 & 1 (cPKU) & & unk & $N R(0)$ & - & \\
\hline $\begin{array}{l}\text { c. }[721 \mathrm{C}>7] ; \\
{\left[931 \_932 \mathrm{del} \mid \mathrm{C} T\right]^{\#}}\end{array}$ & $\begin{array}{l}\text { p. [Arg241Cys]; } \\
\text { [Leu311Glyfs*4] }\end{array}$ & $1(0.38)$ & - & $\begin{array}{l}1(0 / \\
1)\end{array}$ & - & 0 & 1 (mPKU) & & $\mathrm{R}(25)$ & unk & - & \\
\hline c. $[722 \mathrm{G}>\mathrm{A}] ;[722 \mathrm{G}>\mathrm{A}]$ & $\begin{array}{l}\text { p. [Arg241His]; } \\
\text { [Arg241His] }\end{array}$ & $1(0.38)$ & $\begin{array}{l}1(1 / \\
0)\end{array}$ & - & - & 1 (mHP) & 0 & no data & $\mathrm{R}(23)$ & $\mathrm{R}(23)$ & - & \\
\hline c. $[722 \mathrm{G}>\mathrm{A}] ;[782 \mathrm{G}>\mathrm{A}]$ & $\begin{array}{l}\text { p. [Arg241His]; } \\
\text { [Arg261Gln] }\end{array}$ & $1(0.38)$ & $\begin{array}{l}1(1 / \\
0)\end{array}$ & - & - & 1 (mHP) & 0 & no data & $\mathrm{R}(23)$ & $\begin{array}{l}\mathrm{R}^{\mathrm{a}} \\
(38,5)\end{array}$ & - & \\
\hline $\begin{array}{l}\text { c. }[722 \mathrm{G}>\mathrm{A}] ;\left[838 \_842+\right. \\
\text { 3del8] }\end{array}$ & p. [Arg241His]; [His280*] & $1(0.38)$ & $\begin{array}{l}1(1 / \\
0)\end{array}$ & - & - & $1(\mathrm{mHP})$ & 0 & no data & $\mathrm{R}(23)$ & unk & - & NR \\
\hline $\begin{array}{l}\text { c. }[722 \mathrm{G}>\mathrm{A}] ;[842+1 \mathrm{G}> \\
\mathrm{A}]\end{array}$ & p. [Arg241His]; [?] & $1(0.38)$ & - & $\begin{array}{l}1(1 / \\
0)\end{array}$ & - & 1 (mPKU) & 0 & no data & $\mathrm{R}(23)$ & unk & - & \\
\hline $\begin{array}{l}\text { c. }[722 \mathrm{G}>\mathrm{A}] ;[1066-11 \mathrm{G}> \\
\mathrm{A}]\end{array}$ & p. [Arg241His]; [?] & $1(0.38)$ & - & $\begin{array}{l}1(1 / \\
0)\end{array}$ & - & 1 (mPKU) & 0 & no data & $\mathrm{R}(23)$ & NR (0) & - & \\
\hline c. $[727 C>T] ;[727 C>T]$ & p. $\left[\operatorname{Arg} 243^{*}\right] ;\left[\operatorname{Arg} 243^{*}\right]$ & $1(0.38)$ & - & - & $\begin{array}{l}\text { 1) }(0 / \\
1)\end{array}$ & 0 & $1(\mathrm{CPKU})$ & & NR (1) & NR (1) & - & \\
\hline c. $[727 \mathrm{C}>\mathrm{T}] ;[842 \mathrm{C}>\mathrm{T}]$ & p. [Arg243*]; [Pro281Leu] & $1(0.38)$ & - & - & $\begin{array}{l}1(0 / \\
1)\end{array}$ & 0 & 1 (cPKU) & & NR (1) & $N R(1)$ & - & \\
\hline $\begin{array}{l}\text { c. }[727 \mathrm{C}>\mathrm{T}] ;[912+1 \mathrm{G}> \\
\mathrm{A}]\end{array}$ & p. [Arg243*]; [?] & $1(0.38)$ & - & - & $\begin{array}{l}\text { 1) }(0 / \\
1)\end{array}$ & 0 & 1 (cPKU) & & NR (1) & unk & - & \\
\hline c. $[727 C>$ T]; $[1042 C>$ G] & p. [Arg243*]; [Leu348Val] & $1(0.38)$ & - & - & $\begin{array}{l}\text { 1) }(0 / \\
1)\end{array}$ & 0 & 1 (cPKU) & & NR (1) & $\mathrm{R}(41)$ & - & \\
\hline $\begin{array}{l}\text { c. }[727 \mathrm{C}>\mathrm{T}] ;[1066-11 \mathrm{G}> \\
\text { A] }\end{array}$ & p. [Arg243*]; [?] & $1(0.38)$ & - & - & $\begin{array}{l}1(0 / \\
1)\end{array}$ & 0 & 1 (cPKU) & & NR (1) & $\mathrm{NR}(0)$ & - & \\
\hline c. $[727 \mathrm{C}>\mathrm{T}] ;[?]$ & p. [Arg243*]; [?] & $1(0.38)$ & - & - & $\begin{array}{l}1(0 / \\
1)\end{array}$ & 0 & 1 (cPKU) & & NR (1) & - & - & \\
\hline c. $[728 \mathrm{G}>\mathrm{A}] ;$; $[913-7 \mathrm{~A}>\mathrm{G}]$ & p. [Arg243GIn]; [?] & $1(0.38)$ & - & - & - & 0 & 1 (unk) & & R (23) & unk & - & \\
\hline $\begin{array}{l}\text { c. }[728 \mathrm{G}>\mathrm{A}] ;[912+1 \mathrm{G}> \\
\mathrm{A}]\end{array}$ & p. [Arg243GIn]; [?] & $2(0.77)$ & - & - & $\begin{array}{l}\text { 2) }(0 / \\
2)\end{array}$ & 0 & $2(2 \mathrm{cPKU})$ & & $\mathrm{R}(23)$ & unk & - & \\
\hline c. [728G > A]; [1055delG] & $\begin{array}{l}\text { p. [Arg243Gln]; } \\
\text { [Gly352Valfs*48] }\end{array}$ & $2(0.77)$ & - & - & $\begin{array}{l}2(0 / \\
2)\end{array}$ & 0 & $2(2 \mathrm{cPKU})$ & & R (23) & unk & - & NR \\
\hline
\end{tabular}


Table 1 Genotypes observed in French patients with phenylketonuria. Association with clinical phenotypes and response to BH4 loading-test (Continued)

\begin{tabular}{|c|c|c|c|c|c|c|c|c|c|c|c|c|}
\hline c. $[728 \mathrm{G}>\mathrm{A}] ;[1208 \mathrm{C}>\mathrm{T}]$ & $\begin{array}{l}\text { p. [Arg243Gln]; } \\
\text { [Ala403Val] }\end{array}$ & $1(0.38)$ & $\begin{array}{l}1(1 / \\
0)\end{array}$ & - & - & 1 (mHP) & 0 & $6 \mathrm{mHPR}$ & $\mathrm{R}(23)$ & $\mathrm{R}(32)$ & - & \\
\hline $\begin{array}{l}\text { c. }[734 \mathrm{~T}>\mathrm{C}] ;[1315+1 \mathrm{G}> \\
\text { A] }\end{array}$ & p. [Val245Ala]; [?] & $1(0.38)$ & $\begin{array}{l}1(0 / \\
1)\end{array}$ & - & - & 0 & $\begin{array}{l}1 \text { (mHP, HO } 2.4 \mathrm{mg} \\
\% \text {, minus } 4 \%)\end{array}$ & & $\mathrm{R}(50)$ & NR $(0)$ & - & \\
\hline c. $[754 \mathrm{C}>\mathrm{T}] ;[838 \mathrm{G}>\mathrm{A}]$ & $\begin{array}{l}\text { p. [Arg252Trp]; } \\
\text { [Glu280Lys] }\end{array}$ & $1(0.38)$ & - & - & $\begin{array}{l}1(0 / \\
1)\end{array}$ & 0 & 1 (cPKU) & & NR (1) & $\begin{array}{l}\text { NR } \\
(1.95)\end{array}$ & - & \\
\hline $\begin{array}{l}\text { c. [754C > 7]; [838_842+ } \\
\text { 3del8] }\end{array}$ & p. [Arg252Trp]; [His280*] & $1(0.38)$ & - & - & $\begin{array}{l}1(0 / \\
1)\end{array}$ & 0 & $1(\mathrm{CPKU})$ & & NR (1) & unk & - & NR \\
\hline c. $[754 \mathrm{C}>\mathrm{T}] ;[1042 \mathrm{C}>\mathrm{G}]$ & $\begin{array}{l}\text { p. [Arg252Trp]; } \\
\text { [Leu348Val] }\end{array}$ & $1(0.38)$ & - & - & $\begin{array}{l}1(0 / \\
1)\end{array}$ & 0 & 1 (cPKU) & & NR (1) & $\mathrm{R}(41)$ & - & \\
\hline c. $[754 C>T] ;[1045$ T > G] & $\begin{array}{l}\text { p. [Arg252Trp]; } \\
\text { [Ser349Ala] }\end{array}$ & $1(0.38)$ & - & $\begin{array}{l}1(1 / \\
0)\end{array}$ & - & 1 (mPKU) minus $51 \%$ & 0 & no data & NR (1) & unk & - & $\mathrm{R}$ \\
\hline c. [754C > T]; [1055delG] & $\begin{array}{l}\text { p. [Arg252Trp]; } \\
\text { [Gly352Valfs*48] }\end{array}$ & $1(0.38)$ & - & - & $\begin{array}{l}1(0 / \\
1)\end{array}$ & 0 & $1(\mathrm{CPKU})$ & & NR (1) & unk & - & NR \\
\hline $\begin{array}{l}\text { c. }[754 \mathrm{C}>\mathrm{T} ;[1066-11 \mathrm{G}> \\
\text { A] }\end{array}$ & p. [Arg252Trp]; [?] & $1(0.38)$ & - & - & $\begin{array}{l}1(0 / \\
1)\end{array}$ & 0 & $1(\mathrm{CPKU})$ & & NR (1) & NR $(0)$ & - & \\
\hline c. $[754 C>T] ;[1208 C>T]$ & $\begin{array}{l}\text { p. [Arg252Trp]; } \\
\text { [Ala403Val] }\end{array}$ & $1(0.38)$ & - & $\begin{array}{l}1(1 / \\
0)\end{array}$ & - & 1 (mPKU) & 0 & $3 R$ & NR (1) & $\mathrm{R}(32)$ & - & \\
\hline c. $[754 \mathrm{C}>\mathrm{T}] ;[1241 \mathrm{~A}>\mathrm{G}]$ & $\begin{array}{l}\text { p. }[\operatorname{Arg} 252 \operatorname{Trp}] ; \\
\text { [yr414Cys] }\end{array}$ & $1(0.38)$ & - & - & $\begin{array}{l}1(1 / \\
0)\end{array}$ & 1 (cPKU) & 0 & $4 R$ & NR (1) & $R^{a}(36)$ & - & \\
\hline c. $[755 \mathrm{G}>\mathrm{A}] ;[782 \mathrm{G}>\mathrm{A}]$ & $\begin{array}{l}\text { p. }[\operatorname{Arg} 252 \mathrm{Gln}] \\
\text { [Arg261GIn] }\end{array}$ & $1(0.38)$ & - & - & $\begin{array}{l}1(1 / \\
0)\end{array}$ & 1 (cPKU) & 0 & no data & $\begin{array}{l}\text { unk } \\
(3-24)\end{array}$ & $\begin{array}{l}\mathrm{R}^{\mathrm{a}} \\
(38,5)\end{array}$ & - & \\
\hline $\begin{array}{l}\text { c. }[755 \mathrm{G}>\mathrm{A}] ;[\mathrm{[} 066-11 \mathrm{G}> \\
\text { A] }\end{array}$ & p. [Arg252GIn]; [?] & $1(0.38)$ & - & - & $\begin{array}{l}1(0 / \\
1)\end{array}$ & 0 & $1(\mathrm{cPKU})$ & & $\begin{array}{l}\text { unk } \\
(3-24)\end{array}$ & NR $(0)$ & - & \\
\hline c. $[770 \mathrm{G}>\mathrm{A}] ;$; $[932 \mathrm{~T}>\mathrm{C}]$ & $\begin{array}{l}\text { p. [Gly257Asp]; } \\
\text { [Leu311Pro] }\end{array}$ & $1(0.38)$ & - & - & $\begin{array}{l}1(0 / \\
1)\end{array}$ & 0 & $1(\mathrm{CPKU})$ & & unk & NR (1) & - & \\
\hline $\begin{array}{l}\text { c. }[776 \mathrm{C}>\mathrm{T}] ;[1066-11 \mathrm{G}> \\
\text { A] }\end{array}$ & p. [Ala259Val]; [?] & $2(0.77)$ & - & - & $\begin{array}{l}2(0 / \\
2)\end{array}$ & 0 & $2(2 \mathrm{cPKU})$ & & $\begin{array}{l}\text { unk } \\
(2)\end{array}$ & NR $(0)$ & - & \\
\hline c. $[776 C>$ T]; $[1222 C>T]$ & $\begin{array}{l}\text { p. [Ala259Val]; } \\
\text { [Arg408Trp] }\end{array}$ & $2(0.77)$ & - & - & $\begin{array}{l}2(0 / \\
2)\end{array}$ & 0 & $2(2 \mathrm{cPKU})$ & & $\begin{array}{l}\text { unk } \\
(2)\end{array}$ & NR (1) & - & \\
\hline $\begin{array}{l}\text { c. }[776 C>\text { > ]; [970-?_1359 } \\
+ \text { ? ] }]^{3}\end{array}$ & $\begin{array}{l}\text { p. [Ala259Val]; } \\
\text { [Ex10_13del] }\end{array}$ & $1(0.38)$ & - & - & $\begin{array}{l}1(0 / \\
1)\end{array}$ & 0 & $1(\mathrm{cPKU})$ & & $\begin{array}{l}\text { unk } \\
\text { (2) }\end{array}$ & unk & - & \\
\hline c. $[781 C>G]^{\#} ;[913-7 A>G]$ & p. [Arg261Gly]; [?] & $1(0.38)$ & - & - & $\begin{array}{l}1(0 / \\
1)\end{array}$ & 0 & $1(\mathrm{CPKU})$ & & unk & unk & - & \\
\hline c. $[781 C>T] ;[781 C>T]$ & p. $\left[\operatorname{Arg} 261^{*}\right] ;\left[\operatorname{Arg} 261^{*}\right]$ & $1(0.38)$ & - & - & $\begin{array}{l}1(0 / \\
1)\end{array}$ & 0 & $1(\mathrm{CPKU})$ & & NR (1) & NR (1) & - & \\
\hline c. $[781 \mathrm{C}>\mathrm{T}] ;[782 \mathrm{G}>\mathrm{A}]$ & p. [Arg261*]; [Arg261Gln] & $1(0.38)$ & - & - & $\begin{array}{l}1(0 / \\
1)\end{array}$ & 0 & $1(\mathrm{CPKU})$ & & NR (1) & $\begin{array}{l}\mathrm{R}^{\mathrm{a}} \\
(38.5)\end{array}$ & - & \\
\hline c. $[781 C>$ T]; $[1222 C>T]$ & p. [Arg261*]; [Arg408Trp] & $1(0.38)$ & - & - & & 0 & $1(\mathrm{cPKU})$ & & NR (1) & NR (1) & - & \\
\hline
\end{tabular}


Table 1 Genotypes observed in French patients with phenylketonuria. Association with clinical phenotypes and response to BH4 loading-test (Continued)

\begin{tabular}{|c|c|c|c|c|c|c|c|c|c|c|}
\hline & & & & & $\begin{array}{l}\text { 1) }(0 / \\
1)\end{array}$ & & & & & \\
\hline c. $[782 \mathrm{G}>\mathrm{A}] ;[782 \mathrm{G}>\mathrm{A}]$ & $\begin{array}{l}\text { p. }[\operatorname{Arg} 261 \mathrm{Gln}] \\
{[\operatorname{Arg} 261 \mathrm{GIn}]}\end{array}$ & $\begin{array}{l}8 \\
(3.065)\end{array}$ & - & - & $\begin{array}{l}8(6 / \\
2)\end{array}$ & $6(\mathrm{CPKU})$ & $2(\mathrm{CPKU})$ & $\begin{array}{l}59 \mathrm{R} ; \\
11 \mathrm{NR}\end{array}$ & $\begin{array}{l}\mathrm{R}^{\mathrm{a}} \\
(38,5)\end{array}$ & $\begin{array}{l}R^{a} \\
(38,5)\end{array}$ \\
\hline c. $[782 \mathrm{G}>\mathrm{A}] ;[782 \mathrm{G}>\mathrm{C}]$ & $\begin{array}{l}\text { p. }[\text { Arg261GIn]; } \\
\text { [Arg261Pro] }\end{array}$ & $1(0.38)$ & - & - & $\begin{array}{l}\text { 1) }(0 / \\
1)\end{array}$ & 0 & 1 (cPKU) & & $\begin{array}{l}\mathrm{R}^{\mathrm{a}} \\
(38,5)\end{array}$ & unk \\
\hline c. $[782 \mathrm{G}>\mathrm{A}] ;[[814 \mathrm{G}>\mathrm{T}]$ & p. [Arg261GIn]; [Gly272*] & $2(0.77)$ & - & - & $\begin{array}{l}\text { 2) }(0 / \\
2)\end{array}$ & 0 & $2(2 \mathrm{cPKU})$ & & $\begin{array}{l}\mathrm{R}^{\mathrm{a}} \\
(38,5)\end{array}$ & $N R(1)$ \\
\hline c. $[782 \mathrm{G}>\mathrm{A}] ;[838 \mathrm{G}>\mathrm{A}]$ & $\begin{array}{l}\text { p. }[\operatorname{Arg} 261 \mathrm{GIn}] ; \\
\text { [Glu280Lys] }\end{array}$ & $1(0.38)$ & - & - & $\begin{array}{l}\text { 1) }(0 / \\
\text { 1) }\end{array}$ & 0 & 1 (CPKU) & & $\begin{array}{l}\mathrm{R}^{\mathrm{a}} \\
(38,5)\end{array}$ & $\begin{array}{l}\text { NR } \\
(1.95)\end{array}$ \\
\hline $\begin{array}{l}\text { C. }[782 \mathrm{G}>\mathrm{A}] ;[842+1 \mathrm{G}> \\
\mathrm{A}]\end{array}$ & p. [Arg261GIn]; [?] & $2(0.77)$ & - & - & $\begin{array}{l}2(0 / \\
2)\end{array}$ & 0 & $2(2 \mathrm{CPKU})$ & & $\begin{array}{l}\mathrm{R}^{\mathrm{a}} \\
(38,5)\end{array}$ & unk \\
\hline $\begin{array}{l}\text { C. }[782 \mathrm{G}>\mathrm{A}] ;[[912+1 \mathrm{G}> \\
\mathrm{A}]\end{array}$ & p. [Arg261GIn]; [?] & $1(0.38)$ & - & - & $\begin{array}{l}\text { 1) }(0 / \\
\text { 1) }\end{array}$ & 0 & 1 (CPKU) & & $\begin{array}{l}\mathrm{R}^{\mathrm{a}} \\
(38,5)\end{array}$ & unk \\
\hline c. $[782 \mathrm{G}>\mathrm{A}] ;[$ [1045 T > C] & $\begin{array}{l}\text { p. }[\text { Arg261GIn]; } \\
\text { [Ser349Pro] }\end{array}$ & $2(0.77)$ & - & - & $\begin{array}{l}2(1 / \\
1)\end{array}$ & $\begin{array}{l}1(\mathrm{cPKU}) \\
\text { minus } 41 \%\end{array}$ & $\begin{array}{l}1(\mathrm{cPKU}) \text { minus } \\
20 \%\end{array}$ & $3 R$ & $\begin{array}{l}\mathrm{R}^{\mathrm{a}} \\
(38,5)\end{array}$ & NR (1) \\
\hline c. [782G > A]; [1055delG] & $\begin{array}{l}\text { p. [Arg261Gln]; } \\
\text { [Gly352Valfs*48] }\end{array}$ & $1(0.38)$ & - & - & $\begin{array}{l}\text { 1) }(0 / \\
1)\end{array}$ & 0 & 1 (cPKU) & & $\begin{array}{l}\mathrm{R}^{\mathrm{a}} \\
(38,5)\end{array}$ & unk \\
\hline c. $[782 \mathrm{G}>\mathrm{A}] ;[1066-11 \mathrm{G}>\mathrm{A}]$ & p. [Arg261GIn]; [?] & $4(1.53)$ & - & - & $\begin{array}{l}4(0 / \\
4)\end{array}$ & 0 & $4(4 \mathrm{CPKU})$ & & $\begin{array}{l}\mathrm{R}^{\mathrm{a}} \\
(38,5)\end{array}$ & NR (0) \\
\hline c. $[782 \mathrm{G}>\mathrm{A}] ;[[1068 \mathrm{C}>\mathrm{G}]$ & p. [Arg261GIn]; [Tyr356*] & $1(0.38)$ & - & - & $\begin{array}{l}\text { 1) }(0 / \\
\text { 1) }\end{array}$ & 0 & $1(\mathrm{CPKU})$ & & $\begin{array}{l}\mathrm{R}^{\mathrm{a}} \\
(38,5)\end{array}$ & NR (1) \\
\hline c. $[782 \mathrm{G}>\mathrm{A}] ;[1162 \mathrm{G}>\mathrm{A}]$ & $\begin{array}{l}\text { p. [Arg261GIn]; } \\
\text { [Val388Met] }\end{array}$ & $1(0.38)$ & - & - & $\begin{array}{l}\text { 1) }(0 / \\
\text { 1) }\end{array}$ & 0 & $1(\mathrm{CPKU})$ & & $\begin{array}{l}\mathrm{R}^{\mathrm{a}} \\
(38,5)\end{array}$ & $\begin{array}{l}R \\
(72.5)\end{array}$ \\
\hline c. $[782 \mathrm{G}>\mathrm{A}] ;[1208 \mathrm{C}>\mathrm{T}]$ & $\begin{array}{l}\text { p. [Arg261GIn]; } \\
\text { [Ala403Val] }\end{array}$ & $1(0.38)$ & $\begin{array}{l}1(1 / \\
0)\end{array}$ & - & - & $1(\mathrm{mHP})$ & 0 & $6 \mathrm{mHPR}$ & $\begin{array}{l}\mathrm{R}^{\mathrm{a}} \\
(38,5)\end{array}$ & $R(32)$ \\
\hline c. $[782 \mathrm{G}>\mathrm{A}] ;[1222 \mathrm{C}>\mathrm{T}]$ & $\begin{array}{l}\text { p. [Arg261GIn]; } \\
{[\operatorname{Arg} 408 T r p]}\end{array}$ & $4(1.53)$ & - & $\begin{array}{l}1(0 / \\
1)\end{array}$ & $\begin{array}{l}3(0 / \\
3)\end{array}$ & 0 & 4 (1 mPKU, 3 cPKU) & & $\begin{array}{l}\mathrm{R}^{\mathrm{a}} \\
(38,5)\end{array}$ & NR (1) \\
\hline c. $[782 \mathrm{G}>\mathrm{A}] ;[[1241 \mathrm{~A}>\mathrm{G}]$ & $\begin{array}{l}\text { p. }[\operatorname{Arg} 261 \mathrm{Gln}] ; \\
{[\text { Tyr414Cys] }}\end{array}$ & $1(0.38)$ & - & $\begin{array}{l}1(1 / \\
0)\end{array}$ & - & 1 (mPKU) & 0 & $12 \mathrm{R}$ & $\begin{array}{l}\mathrm{R}^{\mathrm{a}} \\
(38,5)\end{array}$ & $\begin{array}{l}R^{a} \\
(38,5)\end{array}$ \\
\hline $\begin{array}{l}\text { C. }[782 G>A] ;[1315+1 G> \\
\text { A] }\end{array}$ & p. [Arg261GIn]; [?] & $\begin{array}{l}5 \\
(1.915)\end{array}$ & - & - & $\begin{array}{l}5(0 / \\
5)\end{array}$ & 0 & $5(5 \mathrm{CPKU})$ & & $\begin{array}{l}\mathrm{R}^{\mathrm{a}} \\
(38,5)\end{array}$ & NR (0) \\
\hline $\begin{array}{l}\text { c. [782G > A]; [510-?-706 } \\
+ \text { ?del] }\end{array}$ & p. [Arg261GIn]; [Ex6del] & $1(0.38)$ & - & - & $\begin{array}{l}\text { 1) }(0 / \\
\text { 1) }\end{array}$ & 0 & $1(\mathrm{CPKU})$ & & $\begin{array}{l}\mathrm{R}^{\mathrm{a}} \\
(38,5)\end{array}$ & unk \\
\hline c. $[782 \mathrm{G}>\mathrm{C}] ;[1241 \mathrm{~A}>\mathrm{G}]$ & $\begin{array}{l}\text { p. [Arg261Pro]; } \\
\text { [Tyr414Cys] }\end{array}$ & $1(0.38)$ & - & - & $\begin{array}{l}1(1 / \\
0)\end{array}$ & 1 (CPKU) & 0 & 2 PKU R; 1 PKU NR & unk & $R^{a}$ (36) \\
\hline $\begin{array}{l}\text { C. }[782 G>C] ;[1315+1 G> \\
\text { A] }\end{array}$ & p. [Arg261Pro]; [?] & $1(0.38)$ & - & - & $\begin{array}{l}\text { 1) }(0 / \\
1)\end{array}$ & 0 & 1 (cPKU) & & unk & NR (0) \\
\hline c. $[812 \mathrm{~A}>\mathrm{G}] ;[1222 \mathrm{C}>\mathrm{T}]$ & & $1(0.38)$ & - & - & & 0 & 1 (cPKU) & & unk & NR (1) \\
\hline
\end{tabular}


Table 1 Genotypes observed in French patients with phenylketonuria. Association with clinical phenotypes and response to BH4 loading-test (Continued)

\begin{tabular}{|c|c|c|c|c|c|c|c|c|c|c|c|}
\hline & $\begin{array}{l}\text { p. [His271Arg]; } \\
\text { [Arg408Trp] }\end{array}$ & & & & $\begin{array}{l}\text { 1(0/ } \\
1)\end{array}$ & & & & & & \\
\hline c. $[814 G>7] ;[1042 C>G]$ & p. [Gly272*]; [Leu348Val] & $1(0.38)$ & - & - & $\begin{array}{l}1(0 / \\
1)\end{array}$ & 0 & $1(\mathrm{cPKU})$ & & NR (1) & $R(41)$ & - \\
\hline c. $[814 G>$ T]; [1045 T>C] & p. [Gly272*]; [Ser349Pro] & $1(0.38)$ & - & - & $\begin{array}{l}\text { 1(0/ } \\
1)\end{array}$ & 0 & 1 (cPKU) & & NR (1) & $N R(1)$ & - \\
\hline $\begin{array}{l}\text { C. }[814 \mathrm{G}>7] ;[1066-11 \mathrm{G}> \\
\text { A] }\end{array}$ & p. [Gly272*]; [?] & $1(0.38)$ & - & - & $\begin{array}{l}1(0 / \\
1)\end{array}$ & 0 & $1(\mathrm{cPKU})$ & & NR (1) & $N R(0)$ & - \\
\hline c. $[814 G>T] ;[1162 G>A]$ & p. [Gly272*]; [Val388Met] & $1(0.38)$ & - & - & $\begin{array}{l}1(1 / \\
0)\end{array}$ & 1 (cPKU) & 0 & no data & NR (1) & $\begin{array}{l}R \\
(27.5)\end{array}$ & - \\
\hline c. $[814 G>7] ;[1169 A>G]$ & p. [Gly272*]; [Glu390Gly] & $2(0.77)$ & - & $\begin{array}{l}2(2 / \\
0)\end{array}$ & - & 2 (mPKU) & 0 & $2 R$ & NR (1) & $\begin{array}{l}R \\
(72.5)\end{array}$ & - \\
\hline c. $[814 G>$ > ]; $[1208 C>T]$ & p. [Gly272*]; [Ala403Val] & $1(0.38)$ & $\begin{array}{l}1(1 / \\
0)\end{array}$ & - & - & 1 (mHP) & 0 & no data & NR (1) & $R(32)$ & - \\
\hline c. $[814 G>T] ;[1222 C>T]$ & p. [Gly272*]; [Arg408Trp] & $1(0.38)$ & - & - & $\begin{array}{l}1(0 / \\
1)\end{array}$ & 0 & $1(\mathrm{cPKU})$ & & NR (1) & $N R(1)$ & - \\
\hline c. $[829 \mathrm{~T}>\mathrm{G}] ;[1042 \mathrm{C}>\mathrm{G}]$ & $\begin{array}{l}\text { p. [Tyr277Asp]; } \\
\text { [Leu348Val] }\end{array}$ & $1(0.38)$ & - & $\begin{array}{l}1(1 / \\
0)\end{array}$ & - & $1(\mathrm{mPKU})$ & 0 & no data & $\begin{array}{l}\text { unk } \\
(0-12)\end{array}$ & $\mathrm{R}(41)$ & - \\
\hline $\begin{array}{l}\text { c. }[829 \mathrm{~T}>\mathrm{G}] ;[1066-11 \mathrm{G}> \\
\text { A] }\end{array}$ & p. [Tyr277Asp]; [?] & $1(0.38)$ & - & - & $\begin{array}{l}\text { 1(0/ } \\
1)\end{array}$ & 0 & 1 (cPKU) & & $\begin{array}{l}\text { unk } \\
(0-12)\end{array}$ & $N R(0)$ & - \\
\hline $\begin{array}{l}\text { c. }[833 C>A] ;[1315+1 G> \\
\text { A] }\end{array}$ & p. [Thr278Asn]; [?] & $1(0.38)$ & - & - & $\begin{array}{l}1(0 / \\
1)\end{array}$ & 0 & $1(\mathrm{cPKU})$ & & unk & $N R(0)$ & - \\
\hline $\begin{array}{l}\text { c. }[837 \mathrm{delC}]^{\#} ;[1315+1 \mathrm{G}> \\
\text { A] }\end{array}$ & p. [His280Asnfs*61]; [?] & $1(0.38)$ & - & - & $\begin{array}{l}\text { 1(0/ } \\
1)\end{array}$ & 0 & 1 (cPKU) & & unk & $N R(0)$ & - \\
\hline c. $[838 \mathrm{G}>\mathrm{A}] ;$; $[838 \mathrm{G}>\mathrm{A}]$ & $\begin{array}{l}\text { p. [Glu280Lys]; } \\
\text { [Glu280Lys] }\end{array}$ & $\begin{array}{l}11 \\
(4.21)\end{array}$ & - & - & $\begin{array}{l}11 \\
(0 / 11)\end{array}$ & 0 & 11 (11 cPKU) & & $\begin{array}{l}\text { NR } \\
(1.95)\end{array}$ & $\begin{array}{l}\text { NR } \\
(1.95)\end{array}$ & - \\
\hline $\begin{array}{l}\text { C. }[838 \mathrm{G}>\mathrm{A}] ;[842+1 \mathrm{G}> \\
\mathrm{A}]\end{array}$ & p. [Glu280Lys]; [?] & $1(0.38)$ & - & - & $\begin{array}{l}1(0 / \\
1)\end{array}$ & 0 & $1(\mathrm{cPKU})$ & & $\begin{array}{l}\text { NR } \\
(1.95)\end{array}$ & unk & - \\
\hline c. $[838 \mathrm{G}>\mathrm{A}] ;\left[869 \mathrm{~A}>\mathrm{T}^{\#}\right.$ & $\begin{array}{l}\text { p. [Glu280Lys]; } \\
\text { [His290Leu] }\end{array}$ & $1(0.38)$ & - & - & $\begin{array}{l}\text { 1(0/ } \\
1)\end{array}$ & 0 & 1 (cPKU) & & $\begin{array}{l}\text { NR } \\
(1.95)\end{array}$ & unk & - \\
\hline c. [838G > A]; [898G > T] & $\begin{array}{l}\text { p. [Glu280Lys]; } \\
\text { [Ala300Ser] }\end{array}$ & $1(0.38)$ & $\begin{array}{l}1(1 / \\
0)\end{array}$ & - & - & $1(\mathrm{mHP})$ & 0 & no data & $\begin{array}{l}\text { NR } \\
(1.95)\end{array}$ & R (31) & - \\
\hline c. [838G > A]; [1055delG] & $\begin{array}{l}\text { p. [Glu280Lys]; } \\
\text { [Gly352Valfs*48] }\end{array}$ & $1(0.38)$ & - & - & $\begin{array}{l}1(0 / \\
1)\end{array}$ & 0 & $1(\mathrm{cPKU})$ & & $\begin{array}{l}\text { NR } \\
(1.95)\end{array}$ & unk & - \\
\hline $\begin{array}{l}\text { c. }[838 \mathrm{G}>\mathrm{A}] ; \text { [ }[1066-11 \mathrm{G}> \\
\mathrm{A}]\end{array}$ & p. [Glu280Lys]; [?] & $1(0.38)$ & - & - & $\begin{array}{l}\text { 1(0/ } \\
1)\end{array}$ & 0 & $1(\mathrm{cPKU})$ & & $\begin{array}{l}\text { NR } \\
(1.95)\end{array}$ & $N R(0)$ & - \\
\hline c. $[838 \mathrm{G}>\mathrm{A}] ;[1066-3 \mathrm{C}>\mathrm{T}]$ & p. [Glu280Lys]; [?] & $2(0.77)$ & - & - & $\begin{array}{l}2(0 / \\
2)\end{array}$ & 0 & 2 (2 cPKU) & & $\begin{array}{l}\text { NR } \\
(1.95)\end{array}$ & $\mathrm{R}$ & - \\
\hline c. [838G > A]; [1208C > T] & & $1(0.38)$ & & - & - & 1 (mHP) & 0 & no data & & $R(32)$ & - \\
\hline
\end{tabular}


Table 1 Genotypes observed in French patients with phenylketonuria. Association with clinical phenotypes and response to BH4 loading-test (Continued)

\begin{tabular}{|c|c|c|c|c|c|c|c|c|c|c|c|c|}
\hline & $\begin{array}{l}\text { p. [Glu280Lys]; } \\
\text { [Ala403Val] }\end{array}$ & & $\begin{array}{l}1(1 / \\
0)\end{array}$ & & & & & & $\begin{array}{l}\mathrm{NR} \\
(1.95)\end{array}$ & & & \\
\hline c. $[838 \mathrm{G}>\mathrm{A}] ;[1222 \mathrm{C}>\mathrm{T}]$ & $\begin{array}{l}\text { p. [Glu280Lys]; } \\
\text { [Arg408Trp] }\end{array}$ & $1(0.38)$ & - & - & $\begin{array}{l}\text { 1) }(0 / \\
1)\end{array}$ & 0 & 1 (cPKU) & & $\begin{array}{l}\mathrm{NR} \\
(1.95)\end{array}$ & NR (1) & - & \\
\hline c. $[838 \mathrm{G}>\mathrm{A}] ;[1243 \mathrm{G}>\mathrm{A}]$ & $\begin{array}{l}\text { p. [Glu280Lys]; } \\
\text { [Asp415Asn] }\end{array}$ & $1(0.38)$ & $\begin{array}{l}1(1 / \\
0)\end{array}$ & - & - & $1(\mathrm{mHP})$ & 0 & no data & $\begin{array}{l}\text { NR } \\
(1.95)\end{array}$ & R (93) & - & \\
\hline $\begin{array}{l}\text { C. }[841 \mathrm{C}>\mathrm{T}] ;[1315+1 \mathrm{G}> \\
\mathrm{A}]\end{array}$ & p. [Pro281Ser]; [?] & $1(0.38)$ & - & - & $\begin{array}{l}1(0 / \\
1)\end{array}$ & 0 & 1 (cPKU) & & unk & NR $(0)$ & - & \\
\hline $\begin{array}{l}\text { c. }[842+1 G>A] ;[1066- \\
11 G>A]\end{array}$ & p. [?]; [?] & $1(0.38)$ & - & - & $\begin{array}{l}1(0 / \\
1)\end{array}$ & 0 & 1 (cPKU) & & unk & NR $(0)$ & - & \\
\hline $\begin{array}{l}\text { c. }[842+3 G>C] ;[842+ \\
3 G>C]\end{array}$ & p. [?]; [?] & $2(0.77)$ & - & - & $\begin{array}{l}\text { 2(0/ } \\
\text { 2) }\end{array}$ & 0 & $2(2 \mathrm{cPKU})$ & & unk & unk & - & \\
\hline c. $[842 C>7] ;[842 C>T]$ & $\begin{array}{l}\text { p. [Pro281Leu]; } \\
\text { [Pro281Leu] }\end{array}$ & $2(0.77)$ & - & - & $\begin{array}{l}\text { 2) }(0 / \\
2)\end{array}$ & 0 & 2 (2 cPKU) & & NR (1) & NR (1) & - & \\
\hline c. $[842 C>T] ;[898 G>T]$ & $\begin{array}{l}\text { p. [Pro281Leu]; } \\
\text { [Ala300Ser] }\end{array}$ & $1(0.38)$ & $\begin{array}{l}1(1 / \\
0)\end{array}$ & - & - & $1(\mathrm{mHP})$ & 0 & $3 R$ & NR (1) & $R(31)$ & - & \\
\hline c. $[842 C>T] ;[926 C>T]$ & p. [Pro281Leu]; [A309Val] & $1(0.38)$ & - & - & $\begin{array}{l}\text { 1)/ } \\
\text { 1) }\end{array}$ & 0 & 1 (cPKU) & & NR (1) & $\mathrm{R}(44)$ & - & \\
\hline c. $[842 \mathrm{C}>$ T]; $[1042 \mathrm{C}>\mathrm{G}]$ & $\begin{array}{l}\text { p. [Pro281Leu]; } \\
\text { [Leu348Val] }\end{array}$ & $2(0.77)$ & - & - & $\begin{array}{l}2(0 / \\
2)\end{array}$ & 0 & $2(2 \mathrm{CPKU})$ & & NR (1) & $\mathrm{R}(41)$ & - & \\
\hline $\begin{array}{l}\text { C. }[842 C>T] ;[1066-11 G> \\
\text { A] }\end{array}$ & p. [Pro281Leu]; [?] & $1(0.38)$ & - & - & $\begin{array}{l}1(0 / \\
1)\end{array}$ & 0 & $1(\mathrm{cPKU})$ & & NR (1) & NR $(0)$ & - & \\
\hline c. $[842 C>T] ;[1222 C>T]$ & $\begin{array}{l}\text { p. [Pro281Leu]; } \\
\text { [Arg408Trp] }\end{array}$ & $1(0.38)$ & - & - & $\begin{array}{l}\text { 1)/ } \\
\text { 1) }\end{array}$ & 0 & 1 (cPKU) & & NR (1) & NR (1) & - & \\
\hline c. $[842 C>$ T]; [1241A > G] & $\begin{array}{l}\text { p. [Pro281Leu]; } \\
\text { [Tyr414Cys] }\end{array}$ & $2(0.77)$ & - & $\begin{array}{l}1(1 / \\
0)\end{array}$ & $\begin{array}{l}1(1 / \\
0)\end{array}$ & 2 (mPKU, cPKU) & 0 & $6 \mathrm{R} ; 1 \mathrm{NR}$ & NR (1) & $R^{a}(36)$ & - & \\
\hline $\begin{array}{l}\text { C. }[842 C>T] ;[1315+1 G> \\
\text { A] }\end{array}$ & p. [Pro281Leu]; [?] & $3(1.15)$ & - & - & $\begin{array}{l}3(1 / \\
2)\end{array}$ & 1 (cPKU) minus $33 \%$ & $2(2 \mathrm{cPKU})$ & $6 \mathrm{CPKUNR}$ & NR (1) & NR (0) & - & \\
\hline c. $[887 \mathrm{~A}>\mathrm{G}]^{\#} ;[1222 \mathrm{C}>\mathrm{T}]$ & $\begin{array}{l}\text { p. [Asp296Gly]; } \\
\text { [Arg408Trp] }\end{array}$ & $1(0.38)$ & - & - & $\begin{array}{l}1(1 / \\
0)\end{array}$ & 1 (cPKU) minus $36 \%$ & 0 & no data & unk & NR (1) & - & unclear \\
\hline c. [896 T > G]; [1222C > T] & $\begin{array}{l}\text { p. [Phe299Cys]; } \\
\text { [Arg408Trp] }\end{array}$ & $1(0.38)$ & - & - & $\begin{array}{l}\text { 1)/ } \\
\text { 1) }\end{array}$ & 0 & 1 (cPKU) & & $\begin{array}{l}\text { unk } \\
\text { (3) }\end{array}$ & NR (1) & - & \\
\hline $\begin{array}{l}\text { c. [896 T > G]; [169-?_352 } \\
\text { + ?del] }\end{array}$ & p. [Phe299Cys]; [Ex3del] & $1(0.38)$ & - & - & $\begin{array}{l}1(0 / \\
1)\end{array}$ & 0 & $1(\mathrm{cPKU})$ & & $\begin{array}{l}\text { unk } \\
\text { (3) }\end{array}$ & unk & - & \\
\hline c. [898G > T]; [1045 T>C] & $\begin{array}{l}\text { p. [Ala300Ser]; } \\
\text { [Ser349Pro] }\end{array}$ & $1(0.38)$ & - & $\begin{array}{l}1(1 / \\
0)\end{array}$ & - & 1 (mPKU) & 0 & $4 R$ & $\mathrm{R}(31)$ & NR (1) & - & \\
\hline $\begin{array}{l}\text { C. }[898 \mathrm{G}>\mathrm{T}] ;[1066-11 \mathrm{G}> \\
\text { A] }\end{array}$ & p. [Ala300Ser]; [?] & $1(0.38)$ & - & $\begin{array}{l}1(1 / \\
0)\end{array}$ & - & 1 (mPKU) & 0 & $16 R$ & $\mathrm{R}(31)$ & NR $(0)$ & - & \\
\hline $\begin{array}{l}\text { C. }[898 \mathrm{G}>\mathrm{T}] ;[1315+1 \mathrm{G}> \\
\text { A] }\end{array}$ & p. [Ala300Ser]; [?] & $2(0.77)$ & $\begin{array}{l}2(2 / \\
0)\end{array}$ & - & - & $2(\mathrm{mHP})$ & 0 & $3 R$ & $\mathrm{R}(31)$ & NR (0) & - & \\
\hline
\end{tabular}


Table 1 Genotypes observed in French patients with phenylketonuria. Association with clinical phenotypes and response to BH4 loading-test (Continued)

\begin{tabular}{|c|c|c|c|c|c|c|c|c|c|c|c|c|c|}
\hline $\begin{array}{l}\text { c. }[912+1 \mathrm{G}>\mathrm{A}] \\
{[1055 \mathrm{del}]}\end{array}$ & p. [?]; [Gly352Valfs*48] & $1(0.38)$ & - & - & $\begin{array}{l}\text { 1) }(0 / \\
\text { 1) }\end{array}$ & 0 & 1 (cPKU) & & unk & unk & - & & NR \\
\hline $\begin{array}{l}\text { c. }[912+1 G>A] ;[1315+ \\
1 G>A]\end{array}$ & p. [?]; [?] & $1(0.38)$ & - & - & $\begin{array}{l}\text { 1 }(0 / \\
1)\end{array}$ & 0 & 1 (cPKU) & & unk & NR (0) & - & & \\
\hline $\begin{array}{l}\text { c. }[912+2 \mathrm{~T}>\mathrm{C}]^{\#} \\
{[1042 \mathrm{C}>\mathrm{G}]}\end{array}$ & p. [?]; [Leu348Val] & $1(0.38)$ & - & - & $\begin{array}{l}\text { 1(0/ } \\
\text { 1) }\end{array}$ & 0 & 1 (cPKU) & & unk & $\mathrm{R}(41)$ & - & & \\
\hline $\begin{array}{l}\text { c. }[913-8 \mathrm{~A}>\mathrm{G}]^{\#} ;[1315+ \\
1 \mathrm{G}>\mathrm{A}]\end{array}$ & p. [?]; [?] & $1(0.38)$ & - & - & $\begin{array}{l}\text { 1) }(0 / \\
1)\end{array}$ & 0 & 1 (cPKU) & & unk & NR (0) & - & & \\
\hline c. $[1042 C>G] ;[1042 C>G]$ & $\begin{array}{l}\text { p. [Leu348Val]; } \\
\text { [Leu348Val] }\end{array}$ & $1(0.38)$ & - & - & $\begin{array}{l}1(1 / \\
0)\end{array}$ & $1(\mathrm{cPKU})$ & 0 & 3 PKU R & $\mathrm{R}(41)$ & $\mathrm{R}(41)$ & - & & \\
\hline $\begin{array}{l}\text { c. }[1042 C>G] ;[1066- \\
11 G>A]\end{array}$ & p. [Leu348Val]; [?] & $1(0.38)$ & - & - & $\begin{array}{l}\text { 1) }(0 / \\
1)\end{array}$ & 0 & $1(\mathrm{cPKU})$ & & $\mathrm{R}(41)$ & NR $(0)$ & - & & \\
\hline c. $[1042 C>G] ;[1169 A>G]$ & $\begin{array}{l}\text { p. [Leu348Val]; } \\
\text { [Glu390Gly] }\end{array}$ & $1(0.38)$ & - & $\begin{array}{l}1(1 / \\
0)\end{array}$ & - & 1 (mPKU) & 0 & $3 \mathrm{R}$ & $R(41)$ & $\begin{array}{l}R \\
(72.5)\end{array}$ & - & & \\
\hline c. $[1042 C>G] ;[1222 C>T]$ & $\begin{array}{l}\text { p. [Leu348Val]; } \\
\text { [Arg408Trp] }\end{array}$ & $1(0.38)$ & - & - & $\begin{array}{l}1(0 / \\
1)\end{array}$ & 0 & 1 (cPKU) & & $\mathrm{R}(41)$ & NR (1) & - & & \\
\hline $\begin{array}{l}\text { c. [1045 T > C]; [1066-3C > } \\
\text { T] }\end{array}$ & p. [Ser349Pro]; [?] & $1(0.38)$ & - & - & $\begin{array}{l}\text { 1(0/ } \\
\text { 1) }\end{array}$ & 0 & 1 (cPKU) & & NR (1) & $\mathrm{R}$ & - & & \\
\hline c. [1045 T > C]; [1162G > A] & $\begin{array}{l}\text { p. [Ser349Pro]; } \\
\text { [Val388Met] }\end{array}$ & $2(0.77)$ & - & - & $\begin{array}{l}2(1 / \\
1)\end{array}$ & 1 (cPKU) minus $72 \%$ & $\begin{array}{l}1(\mathrm{CPKU}) \text { minus } \\
20 \%\end{array}$ & $2 \mathrm{R} ; 4$ CPKU NR & NR (1) & $\begin{array}{l}\mathrm{R} \\
(27.5)\end{array}$ & - & & \\
\hline c. [1045 T > C]; [1169A > G] & $\begin{array}{l}\text { p. [Ser349Pro]; } \\
\text { [Glu390Gly] }\end{array}$ & $1(0.38)$ & - & $\begin{array}{l}1(1 / \\
0)\end{array}$ & - & 1 (mPKU) & 0 & $5 R$ & NR (1) & $\begin{array}{l}\mathrm{R} \\
(72.5)\end{array}$ & - & & \\
\hline c. $[1045 \mathrm{~T}>\mathrm{C}] ;[1243 \mathrm{G}>\mathrm{A}]$ & $\begin{array}{l}\text { p. [Ser349Pro]; } \\
\text { [Asp415Asn] }\end{array}$ & $1(0.38)$ & $\begin{array}{l}1(0 / \\
1)\end{array}$ & - & - & 0 & $1(\mathrm{mHP})$ & & NR (1) & $\mathrm{R}(93)$ & - & & \\
\hline $\begin{array}{l}\text { c. [1045 T > C]; [442-?_509 } \\
\text { +?del] }\end{array}$ & p. [Ser349Pro]:Ex5del] & $1(0.38)$ & - & - & $\begin{array}{l}1(0 / \\
1)\end{array}$ & 0 & 1 (cPKU) & & NR (1) & unk & - & & \\
\hline $\begin{array}{l}\text { c. [1045 T> G]; [1045 T> } \\
\text { G] }\end{array}$ & $\begin{array}{l}\text { p. [Ser349Ala]; } \\
\text { [Ser349Ala] }\end{array}$ & $2(0.77)$ & - & $\begin{array}{l}1(1 / \\
0)\end{array}$ & $\begin{array}{l}1(0 / \\
1)\end{array}$ & 1 (mPKU) minus $71.5 \%$ & 1 (cPKU) minus $7 \%$ & $1 \mathrm{R}$ & unk & unk & - & R & $\mathrm{R}$ \\
\hline c. [1055delG]; [1055delG] & $\begin{array}{l}\text { p. [Gly352Valfs*48]; } \\
\text { [Gly352Valfs*48] }\end{array}$ & $7(2.68)$ & - & - & $\begin{array}{l}7(0 / \\
7)\end{array}$ & 0 & 7 (7 cPKU) & & unk & unk & - & $N R$ & NR \\
\hline $\begin{array}{l}\text { c. [1055delG]; [1066-11G > } \\
\text { A] }\end{array}$ & p. [Gly352Valfs*48]; [?] & $2(0.77)$ & - & - & $\begin{array}{l}2(0 / \\
2)\end{array}$ & 0 & $2(2 \mathrm{cPKU})$ & & unk & NR $(0)$ & - & $N R$ & \\
\hline c. [1055delG]; [1208C > T] & $\begin{array}{l}\text { p. [Gly352Valfs*48]; } \\
\text { [Ala403Val] }\end{array}$ & $1(0.38)$ & $\begin{array}{l}1(1 / \\
0)\end{array}$ & - & - & $1(\mathrm{mHP})$ & 0 & no data & unk & $\mathrm{R}(32)$ & - & NR & \\
\hline $\begin{array}{l}\text { c. }[1065+1 G>A] ; \\
{[1169 A>G]}\end{array}$ & p. [?]; [Glu390Gly] & $1(0.38)$ & $\begin{array}{l}1(1 / \\
0)\end{array}$ & - & - & $1(\mathrm{mHP})$ & 0 & no data & unk & $\begin{array}{l}\mathrm{R} \\
(72.5)\end{array}$ & - & & \\
\hline \multirow[t]{2}{*}{$\begin{array}{l}\text { c. }[1066-2 A>T]^{\#} ;[1169 A> \\
\text { G] }\end{array}$} & p. [Glu390Gly]; [?] & $1(0.38)$ & - & - & - & 1 ('unk) & 0 & no data & unk & $\begin{array}{l}\mathrm{R} \\
(72.5)\end{array}$ & - & & \\
\hline & p. [?]; [?] & & - & & & 1 (cPKU) minus $31 \%$ & 4 (1 mPKU, 3 cPKU) & $10 \mathrm{R} ; 74 \mathrm{NR}$ & NR (0) & NR (0) & - & & \\
\hline
\end{tabular}


Table 1 Genotypes observed in French patients with phenylketonuria. Association with clinical phenotypes and response to BH4 loading-test (Continued)

\begin{tabular}{|c|c|c|c|c|c|c|c|c|c|c|c|c|}
\hline $\begin{array}{l}\text { c. }[1066-11 G>A] ;[1066- \\
11 G>A]\end{array}$ & & $\begin{array}{l}5 \\
(1.915)\end{array}$ & & $\begin{array}{l}\text { 1) }(0 / \\
1)\end{array}$ & $\begin{array}{l}4(1 / \\
3)\end{array}$ & & & & & & & \\
\hline $\begin{array}{l}\text { c. }[1066-11 \mathrm{G}>\mathrm{A}] \\
{[1162 \mathrm{G}>\mathrm{A}]}\end{array}$ & p. [?]; [Val388Met] & $1(0.38)$ & - & - & $\begin{array}{l}\text { 1)/ } \\
\text { 1) }\end{array}$ & 0 & 1 (cPKU) & & NR (0) & $\begin{array}{l}R \\
(27.5)\end{array}$ & - & \\
\hline $\begin{array}{l}\text { c. }[1066-11 \mathrm{G}>\mathrm{A}] ; \\
{[1196 \mathrm{~T}>\mathrm{C}]^{\#}}\end{array}$ & p. [?]; [Val399Ala] & $1(0.38)$ & - & - & $\begin{array}{l}1(1 / \\
0)\end{array}$ & 1 (cPKU) minus $97 \%$ & 0 & no data & NR $(0)$ & unk & - & $\mathrm{R}$ \\
\hline $\begin{array}{l}\text { c. }[1066-11 G>A] ;[1199+ \\
1 G>C]\end{array}$ & p. [?]; [?] & $3(1.15)$ & - & $\begin{array}{l}\text { 1 }(0 / \\
1)\end{array}$ & $\begin{array}{l}2(1 / \\
1)\end{array}$ & 1 (cPKU) minus $37.5 \%$ & 2 (mPKU, cPKU) & no data & NR $(0)$ & unk & - & unclear \\
\hline $\begin{array}{l}\text { c. }[1066-11 G>A] ; \\
{[1222 C>T]}\end{array}$ & p. [?]; [Arg408Trp] & $1(0.38)$ & - & - & $\begin{array}{l}\text { 1) }(0 / \\
1)\end{array}$ & 0 & $1(\mathrm{cPKU})$ & & NR (0) & NR (1) & - & \\
\hline $\begin{array}{l}\text { c. }[1066-11 G>A] ; \\
{[1223 G>A]}\end{array}$ & p. [?]; [Arg408GIn] & $1(0.38)$ & - & - & $\begin{array}{l}1(1 / \\
0)\end{array}$ & 1 (cPKU) & 0 & no data & NR $(0)$ & $\begin{array}{l}R \\
(49.7)\end{array}$ & - & \\
\hline $\begin{array}{l}\text { c. }[1066-11 G>A] \\
{[1241 A>G]}\end{array}$ & p. [?]; [Tyr414Cys] & $4(1.53)$ & - & $\begin{array}{l}4(3 / \\
1)\end{array}$ & - & 3 (mPKU) & $\begin{array}{l}1 \text { (mPKU) minus } \\
21 \%\end{array}$ & $18 \mathrm{R} ; 2 \mathrm{NR}$ & NR (0) & $R^{a}(36)$ & - & \\
\hline $\begin{array}{l}\text { c. }[1066-11 \mathrm{G}>\mathrm{A}] ;[1315+ \\
1 \mathrm{G}>\mathrm{A}]\end{array}$ & p. [?]; [?] & $3(1.15)$ & - & - & $\begin{array}{l}3(0 / \\
3)\end{array}$ & 0 & 3 (3 cPKU) & & NR (0) & NR (0) & - & \\
\hline $\begin{array}{l}\text { c. [1066-11G > A]; [510- } \\
\text { ?_706+?dup] }]^{\#}\end{array}$ & p. [?]; [Ex6dup] & $1(0.38)$ & - & $\begin{array}{l}\text { 1(0/ } \\
\text { 1) }\end{array}$ & - & 0 & 1 (mPKU) & & NR $(0)$ & unk & - & \\
\hline c. [1066-11G > A]; [?] & p. [?]; [?] & $1(0.38)$ & - & - & $\begin{array}{l}\text { 1 }(0 / \\
1)\end{array}$ & 0 & $1(\mathrm{cPKU})$ & & NR $(0)$ & - & - & \\
\hline $\begin{array}{l}\text { C. }[1066-3 C>\text { T]; [1180G > } \\
\left.T^{\#}\right]^{\#}\end{array}$ & p. [?]; [Asp394Tyr] & $1(0.38)$ & - & $\begin{array}{l}\text { 1) }(0 / \\
1)\end{array}$ & - & 0 & 1 (mPKU) & & $\mathrm{R}$ & unk & - & \\
\hline c. $[1068 \mathrm{C}>\mathrm{G}] ;$ [1162G > A] & p. [Tyr356*]; [Val388Met] & $1(0.38)$ & - & - & $\begin{array}{l}1(1 / \\
0)\end{array}$ & 1 (cPKU) & 0 & no data & NR (1) & $\begin{array}{l}\mathrm{R} \\
(27.5)\end{array}$ & - & \\
\hline c. $[1139 C>T] ;[1180 G>]^{\#}$ & $\begin{array}{l}\text { p. [Thr380Met]; } \\
\text { [Asp394Tyr] }\end{array}$ & $1(0.38)$ & - & $\begin{array}{l}1(1 / \\
0)\end{array}$ & - & $1(\mathrm{mPKU})$ & 0 & no data & $\mathrm{R}$ & unk & - & \\
\hline $\begin{array}{l}\text { c. }[1139 \mathrm{C}>\text { T]; [1-?_60 } \\
\text { + ?del] }{ }^{\#}\end{array}$ & p. [Thr380Met]; [Ex1del] & $1(0.38)$ & $\begin{array}{l}1(1 / \\
0)\end{array}$ & - & - & $1(\mathrm{mHP})$ & 0 & no data & $\mathrm{R}$ & unk & - & \\
\hline c. $[1162 \mathrm{G}>\mathrm{A}] ;[1162 \mathrm{G}>\mathrm{A}]$ & $\begin{array}{l}\text { p. [Val388Met]; } \\
\text { [Val388Met] }\end{array}$ & $1(0.38)$ & - & - & $\begin{array}{l}\text { 1) }(0 / \\
1)\end{array}$ & 0 & 1 (cPKU) & & $\begin{array}{l}\mathrm{R} \\
(27.5)\end{array}$ & $\begin{array}{l}\mathrm{R} \\
(27.5)\end{array}$ & - & \\
\hline c. $[1162 \mathrm{G}>\mathrm{A}] ;[1169 \mathrm{~A}>\mathrm{G}]$ & $\begin{array}{l}\text { p. [Val388Met]; } \\
\text { [Glu390Gly] }\end{array}$ & $1(0.38)$ & - & $\begin{array}{l}1(1 / \\
0)\end{array}$ & - & 1 (mPKU) & 0 & $8 R$ & $\begin{array}{l}\mathrm{R} \\
(27.5)\end{array}$ & $\begin{array}{l}\mathrm{R} \\
(72.5)\end{array}$ & - & \\
\hline c. $[1162 \mathrm{G}>\mathrm{A}] ;[1223 \mathrm{G}>\mathrm{A}]$ & $\begin{array}{l}\text { p. [Val388Met]; } \\
\text { [Arg408GIn] }\end{array}$ & $1(0.38)$ & - & - & $\begin{array}{l}1(1 / \\
0)\end{array}$ & 1 (cPKU) & 0 & no data & $\begin{array}{l}\mathrm{R} \\
(27.5)\end{array}$ & $\begin{array}{l}\mathrm{R} \\
(49,7)\end{array}$ & - & \\
\hline $\begin{array}{l}\text { C. }[1163 \mathrm{~T}>\mathrm{C}] \text {; }[\text { [1208C > } \\
\text { T] }\end{array}$ & $\begin{array}{l}\text { p. Val388Ala]; } \\
\text { [Ala403Val] }\end{array}$ & $1(0.38)$ & - & $\begin{array}{l}1(1 / \\
0)\end{array}$ & - & 1 (mPKU) & 0 & no data & unk & $R(32)$ & - & \\
\hline c. $[1169 \mathrm{~A}>\mathrm{G}] ;[1241 \mathrm{~A}>\mathrm{G}]$ & $\begin{array}{l}\text { p. [Glu390Gly]; } \\
\text { [Tyr414Cys] }\end{array}$ & $1(0.38)$ & $\begin{array}{l}1(1 / \\
0)\end{array}$ & - & - & $1(\mathrm{mHP})$ & 0 & no data & $\begin{array}{l}R \\
(72.5)\end{array}$ & $R^{a}(36)$ & - & \\
\hline c. [1169A > G]; [?] & p. [Glu390Gly]; [?] & $1(0.38)$ & & - & - & $1(\mathrm{mHP})$ & 0 & & & - & - & \\
\hline
\end{tabular}


Table 1 Genotypes observed in French patients with phenylketonuria. Association with clinical phenotypes and response to BH4 loading-test (Continued)

\begin{tabular}{|c|c|c|c|c|c|c|c|c|c|c|c|c|}
\hline & & & $\begin{array}{l}1(1 / \\
0)\end{array}$ & & & & & & $\begin{array}{l}R \\
(72.5)\end{array}$ & & & \\
\hline $\begin{array}{l}\text { c. }[1199+1 G>C] ;[1199+ \\
1 G>C]\end{array}$ & p. [?]; [?] & $1(0.38)$ & - & - & $\begin{array}{l}1(0 / \\
1)\end{array}$ & 0 & 1 (cPKU) & & unk & unk & - & \\
\hline c. $[1208 C>$ T]; $[1222 C>T]$ & $\begin{array}{l}\text { p. [Ala403Val]; } \\
\text { [Arg408Trp] }\end{array}$ & $2(0.77)$ & $\begin{array}{l}1(1 / \\
0)\end{array}$ & $\begin{array}{l}1(1 / \\
0)\end{array}$ & - & $2(\mathrm{mHP}, \mathrm{mPKU})$ & 0 & $6 \mathrm{R}$ & $\mathrm{R}(32)$ & $N R(1)$ & - & \\
\hline $\begin{array}{l}\text { c. }[1208 C>T] ;[1315+ \\
1 G>A]\end{array}$ & p. [Ala403Val]; [?] & $1(0.38)$ & $\begin{array}{l}1(1 / \\
0)\end{array}$ & - & - & $1(\mathrm{mHP})$ & 0 & $9 \mathrm{R}$ & R (32) & $N R(0)$ & - & \\
\hline c. $[1222 C>T] ;[1222 C>T]$ & $\begin{array}{l}\text { p. [Arg408Trp]; } \\
\text { [Arg408Trp] }\end{array}$ & $\begin{array}{l}5 \\
(1.915)\end{array}$ & - & - & $\begin{array}{l}5(0 / \\
5)\end{array}$ & 0 & 5 (5 cPKU) & & NR (1) & $N R(1)$ & - & \\
\hline c. $[1222 C>T] ;[1223 G>A]$ & $\begin{array}{l}\text { p. [Arg408Trp]; } \\
\text { [Arg408GIn] }\end{array}$ & $1(0.38)$ & - & $\begin{array}{l}1(1 / \\
0)\end{array}$ & - & 1 (mPKU) & 0 & no data & NR (1) & $\begin{array}{l}R \\
(49.7)\end{array}$ & - & \\
\hline c. $[1222 C>$ T]; $[1241 \mathrm{~A}>\mathrm{G}]$ & $\begin{array}{l}\text { p. }[\text { Arg408Trp]; } \\
\text { [yr414Cys] }\end{array}$ & $\begin{array}{l}5 \\
(1.915)\end{array}$ & - & $\begin{array}{l}4(2 / \\
2)\end{array}$ & $\begin{array}{l}\text { 1) }(0 / \\
1)\end{array}$ & $\begin{array}{l}2(\mathrm{mPKU}) \text { minus } 43 \%, \\
\text { minus } 66 \%\end{array}$ & $3(2 \mathrm{mPKU}, 1 \mathrm{cPKU})$ & $30 \mathrm{R} ; 6 \mathrm{NR}$ & NR (1) & $R^{a}(36)$ & - & \\
\hline $\begin{array}{l}\text { c. }[1222 C>T] ;[1315+ \\
1 G>A]\end{array}$ & p. [Arg408Trp]; [?] & $3(1.15)$ & - & - & $\begin{array}{l}3(0 / \\
3)\end{array}$ & 0 & $3(3 \mathrm{cPKU})$ & & NR (1) & $N R(0)$ & - & \\
\hline c. [1222C > T]; [?] & p. [Arg408Trp]; [?] & $1(0.38)$ & - & $\begin{array}{l}1(0 / \\
1)\end{array}$ & - & 0 & $1(\mathrm{mPKU})$ & & NR (1) & - & - & \\
\hline c. $[1223 \mathrm{G}>\mathrm{A}] ;[1241 \mathrm{~A}>\mathrm{G}]$ & $\begin{array}{l}\text { p. }[\operatorname{Arg} 408 \mathrm{GIn}] \\
\text { [yr414Cys] }\end{array}$ & $2(0.77)$ & $\begin{array}{l}1(1 / \\
0)\end{array}$ & - & $\begin{array}{l}1(1 / \\
0)\end{array}$ & $2(\mathrm{mHP}, \mathrm{cPKU})$ & 0 & $6 R$ & $\begin{array}{l}R \\
(49.7)\end{array}$ & $\begin{array}{l}R^{a} \\
(38,5)\end{array}$ & - & \\
\hline $\begin{array}{l}\text { c. }[1241 \mathrm{~A}>\mathrm{G}] ;[1315+ \\
1 \mathrm{G}>\mathrm{A}]\end{array}$ & p. [Tyr414Cys]; [?] & $3(1.15)$ & - & $\begin{array}{l}1(1 / \\
0)\end{array}$ & $\begin{array}{l}2(1 / \\
1)\end{array}$ & $\begin{array}{l}2 \text { (mPKU, cPKU) minus } \\
34 \% \text { minus } 39 \%\end{array}$ & $\begin{array}{l}1(\mathrm{CPKU}) \text { minus } \\
21 \%\end{array}$ & 24 PKU R; 1 PKU NR & $\begin{array}{l}R^{a} \\
(36)\end{array}$ & $N R(0)$ & - & \\
\hline $\begin{array}{l}\text { c. [1241A > G]; [169-?_352 } \\
\text { +?del] }\end{array}$ & p. [Tyr414Cys]; [Ex3del] & $1(0.38)$ & - & - & $\begin{array}{l}\text { 1) }(0 / \\
1)\end{array}$ & 0 & 1 (cPKU) & & $\begin{array}{l}R^{a} \\
(36)\end{array}$ & unk & - & \\
\hline $\begin{array}{l}\text { c. [1243G > A]; [169-?_352 } \\
\text { +?del] }\end{array}$ & p. [Asp415Asn]; [Ex3del] & $1(0.38)$ & - & $\begin{array}{l}1(1 / \\
0)\end{array}$ & - & 1 (mPKU) & 0 & no data & R (93) & unk & - & \\
\hline $\begin{array}{l}\text { c. }[1249 \mathrm{~T}>\mathrm{A}] ;[1315+ \\
1 \mathrm{G}>\mathrm{A}]\end{array}$ & p. [Tyr417Asn]; [?] & $1(0.38)$ & - & $\begin{array}{l}1(1 / \\
0)\end{array}$ & - & 1 (mPKU) minus $37 \%$ & 0 & no data & unk & $N R(0)$ & - & unclear \\
\hline $\begin{array}{l}\text { c. }[1315+1 \mathrm{G}>\mathrm{A}] ; \text { [1-?_60 } \\
+ \text { ? dell }]^{\#}\end{array}$ & p. [?]; [Ex1del] & $1(0.38)$ & - & - & $\begin{array}{l}\text { 1) }(0 / \\
1)\end{array}$ & 0 & 1 (cPKU) & & NR $(0)$ & unk & - & \\
\hline c. [?]; [?] & p. [?]; [?] & $1(0.38)$ & - & - & $\begin{array}{l}1(0 / \\
1)\end{array}$ & 0 & 1 (cPKU) & & - & - & - & \\
\hline
\end{tabular}


thought moderate, in their Phe concentration (minus $31 \%$ and minus $33 \%)$.

Confrontation of genotypes and BH4-loading tests concluded that only patients with mHP carrying 2 responsive mutations are always BH4-responders. Response to $\mathrm{BH} 4$ cannot be predicted in other patients carrying either two or one responsive mutations. However, the milder the phenotype is, the most probable the $\mathrm{BH} 4$ test is positive.

\section{Associations of response to $\mathrm{BH} 4-$ loading test with type and location of mutations}

Any mutation other than missense mutation is predictive of a non-response. Indeed, first, $98.2 \%$ of responsive patients carried at least one missense mutation. Second, frequency of BH4-responders was higher in carriers of 2 missense mutations (45.2\%) than in carriers of 1 missense mutation combined with another type of mutations - such as frameshift, nonsense or splice-site mutation (29.0\%) [Fisher exact test, see Additional file 5: Figure S5]. Third, genotypes combining frameshift, in-frame, nonsense and splice-site mutations were non-responsive, except in two cases where combinations of 2 splice site mutations gave positive - though moderate - responses (minus $31 \%$ and $37.5 \%$ ).

We looked for a possible link between BH4responsiveness and the localisation of mutations. The oligomerization domain seems highly relevant to responsiveness since more responders were found in carriers of one missense mutation in the oligomerization domain $(71.4 \%)$ than in carriers of one missense mutation in the catalytic $(31.4 \%)$ or regulatory $(27.2 \%)$ domains $(p=0.0006$; Fisher exact test; [see Additional file 6: Figure S6]). In addition, even in a single domain there are differences between exons. Thus, more BH4responders were found in carriers of missense mutations in exon 11, 12 or 8 while carriers of missense mutations in exon 7 were more often BH4-unresponsive (Fisher exact test; [Additional file 7: Figure S7]).

\section{Discussion}

In the present study, patients were classified according to pre-treatment Phe concentration following French and European recommendations into three phenotype categories i.e. mHP (180-600 $\mu \mathrm{mol} / \mathrm{L})$, mPKU (600$1200 \mu \mathrm{mol} / \mathrm{L})$ and cPKU (>1200 $\mu \mathrm{mol} / \mathrm{L}$ Phe) $[5,22]$. While recent European consensus publication is still pending, NIH American conference consensus, which was published at a time when our study was completed, proposed 5 categories, namely $\mathrm{mHP}$ not requiring treatment $(<360 \mu \mathrm{mol} / \mathrm{L})$, mild HP-gray zone (360-600 $\mu \mathrm{mol} / \mathrm{L})$, mPKU $(600-900 \mu \mathrm{mol} / \mathrm{L})$, moderate PKU (900-1200 $\mu \mathrm{mol} / \mathrm{L})$ and $\mathrm{cPKU}(>1200 \mu \mathrm{mol} / \mathrm{L}$ Phe) [26]. This different classification system has to be kept in mind when considering the findings and conclusions in our mHP group.

Our multicentric cohort study represents respectively $17.4 \%$ of global cPKU and mPKU and $2.6 \%$ of global $\mathrm{mHP}$ diagnosed in France since the beginning of the neonatal screening in the seventies (data from the French neonatal screening association AFDPHE, [27]). In this study, in which we included only 364 patients with both genetic testing and $\mathrm{BH} 4$ testing, frequency of $\mathrm{mHP}$ is $9 \%$. Frequency of $\mathrm{mHP}$ in all genotyped individuals $(n=701)$ in our center is $19.3 \%$, which is higher than the frequency in the 364 patients included in this study (9\%), but still not representative of the $39.4 \%$ of mHP observed among the whole PKU patients screened in metropolitan France since the beginning of the neonatal screening ([27]. Underrepresentation of patients with the milder phenotypes can be partly explained by the fact that there is no recommendation for BH4-testing in patients with Phe concentration under $480 \mu \mathrm{mol} / \mathrm{L}$. Several mHP are lost to follow-up, in a center dependent manner, and they miss either molecular analysis and/ or BH4 testing.

Investigation of the $P A H$ gene combining $\mathrm{dHPLC}$, sequencing and MLPA allowed us to reach a $99.05 \%$ mutation detection rate, in agreement with results found in Australia: $99.0 \%$ [28], Austria: $98.6 \%$, [29] or Turkey: $97.3 \%$ [30]. dHPLC is a useful method for screening a large set of samples. It proved to be reliable in our hands and cost effective by narrowing down the number of exons needed to be sequenced. However, because of the chemistry of the method, the genetic variants may not be clearly separated. Now several factors argue that direct sequencing should be the first-line test to identify mutations in the $P A H$ gene. Sequencing is carried out faster and costs have dropped down. In addition, next generation sequencing is spreading quickly and allows analyzing a large number of patients in a short time and at costs going lower and lower. Next generation sequencing also allows analyzing the whole gene including introns and regulatory regions. A spectrum of 127 mutations is reported in this study, including $82 \mathrm{mu}$ tations with unknown BH4-responsiveness, according to information available in the literature. This study enables us to classify 5 of them as responsive (c.434A $>\mathrm{T}$, c.500A > T, c.529G > C, c.1045 T> G and c.1196 T > C). The mutation c.1196 T $>\mathrm{C}$ (p.Val399Ala) is positioned four nucleotides upstream the exon/intron junction in exon 11 and one base upstream the 5' splice-site. Exon 11 is particularly vulnerable to mutations affecting splicing regulatory elements [31]. Thus, c.1197A > T and c.1199G $>\mathrm{A}$ have been shown to lead to exon skipping [32]. Minigene assays are needed to determine if the mutation c.1196 T > C could or not disrupt the balance between exonic splicing enhancers and silencers [31]. 
The c.434A $>\mathrm{T}$ (p.Asp145Val) mutation involves the substitution of a charged amino acid by a hydrophobic residue. The c.500A > T (p.Asn167lle) mutation involves the substitution of a polar residue by a hydrophobic residue. The c.529G > C (p.Val177Leu) mutation leads to the substitution of a hydrophobic residue, mostly buried in the loop between C2 and C3 alpha helices [33], by a residue with similar physical and chemical properties. Another mutation affecting the same nucleotide, c.529G > A (p.Val177Met), had previously been described as BH4-responsive [10]. Residue Ser349 is an essential amino acid in the active site of the gene. The substitution of Serine349 by Alanine (c.1045 T > G) removes the hydroxyl group within hydrogen bonding distance of the iron-chelating $\mathrm{H} 285$ required for $\mathrm{PAH}$ catalytic activity. Missense mutations leading to modifications of either the protein overall hydrophobicity or the ability to form hydrogen bonds within the polypeptide backbone or between side chains may impact on the folding/conformation of PAH. Gersting and colleagues suggested that the consequences of these missense mutations might be compensated for by pharmacological doses of BH4 [7]. However, further functional studies are needed to verify that restoration of the enzymatic activity is possible by the addition of BH4 [7, 34, 35]. Another group of five mutations fitted the criteria for classification as responsive mutations but were not recognized as such. Indeed, there were observed in patients with a BH4-responsive rate comprised between 30 and 40 percent and we do believe that there responsiveness should be subsequently assessed by the long-term achievement of blood Phe target concentration under $\mathrm{BH} 4$ treatment.

The position and the nature of the mutation influence the activity of $\mathrm{PAH}$ enzyme, which in turn determines the patient phenotype [21]. Our study suggests that for any genotype to be potentially $\mathrm{BH} 4$-responsive it needs to contain one missense mutation. Indeed, $98.2 \%$ of the responsive patients in this study carried at least one missense mutation. Conversely, homozygous or compound heterozygous patients carrying any other type of mutations are likely not to be BH4-responders, and administering a long term BH4-therapy to them will not yield an appropriate cost-effectiveness ratio. It is true that combinations of some splice-site mutations were reported previously to be associated with a partial BH4-responder phenotype. The explanation was that those mutations were not fully penetrant, determining a phenotype with persistent residual activity [36]. However, for these patients, based on our experience, $\mathrm{BH} 4$ would have rather limited effect in improving dietary Phe tolerance.

In this study, the frequency of $\mathrm{BH} 4$-responders reached $31.6 \%$. It is of note that, if the threshold of responsiveness was more severe (decrease of Phe concentration >40\%), the overall responsive rate would be $22.9 \%$, which is actually a more reasonable estimate of patients treated with
BH4 in France (139 patients: data may 2013). Zurfluh and colleagues [10] postulated that the average frequency of patients potentially BH4-responsive in European populations is $55 \%$. However, these authors considered the BH4responsive mutations to be dominant in compound heterozygous patients and classified carriers of one BH4responsive allele as systematically responsive. This is not always true. Two mutant monomers interact to constitute the holoenzyme and there may be unexpected negative as well as positive inter-allelic complementation in compound heterozygous [4]. Indeed, we found that genotypes carrying 1 or 2 responsive mutations cannot be expected to be always BH4-responsive. Actually, only $66.7 \%$ of patients carrying 2 responsive mutations, $35.4 \%$ of patients carrying 1 responsive mutation and 1 nonresponsive mutation and $35.2 \%$ of patients carrying 1 responsive mutation and 1 unclassified mutation were effective BH4-responders. It must be stressed out that BH4-responsiveness depends on the disease phenotype, since $100 \%$ of patients with $\mathrm{mHP}$ carrying two responsive alleles were found to be BH4-responders whereas this frequency was only $42.3 \%$ for patients with cPKU.

Fourteen genotypes were assigned to both responsive and non-responsive patients. Similar discordances involving some of these genotypes were already described in the BioPKU database [2], as reported in Table 1. Indeed, while the c. [1066-11G > A]; [1066-11G > A] genotype, associated with a null predicted residual activity, was observed in five PKU non-responsive patients, one tPKU patient carrying this genotype showed a positive $\mathrm{BH} 4$ responsive test (H0 $1356 \mu \mathrm{mol} / \mathrm{L})$. However, this patient showed a Phe decrease limited to $31 \%$. A second example is c. [194 T > C]; [1222C > T] (p.[Ile65Thr]; [Arg408Trp]) genotype, associated both with one non-responsive tPKU (H0 $2760 \mu \mathrm{mol} / \mathrm{L})$ and one responsive tPKU (H0 $1380 \mu \mathrm{mol} / \mathrm{L}$ ) with a Phe decrease limited to $32 \%$. This dual BH4-responsiveness may be due to a BH4loading test performed under a rather strict low-Phe diet with subnormal Phe concentrations. Indeed, in the case of some mutations, the stimulation effect of $\mathrm{BH} 4$ on residual enzymatic activity is weaker at low Phe concentrations, as previously described for c.782G > A (p.Arg261Gln) $[4,9,34]$. In our study, patients with p.[Arg261Gln]; [Arg261Gln] genotype and low Phe concentrations at the beginning of $\mathrm{BH} 4$-loading test were found to be BH4-unresponsive whereas patients with the same genotype but higher Phe concentrations were found to be responsive (Phe decrease of minus $66 \%$, minus $59 \%$, minus $38 \%$, minus $37 \%$ and $0 \%$ for basal Phe concentration before testing of 1080, 1236, 768, 960 and $430 \mu \mathrm{mol} / \mathrm{L}$ respectively). Another reason for dual responsiveness to $\mathrm{BH} 4$ is the intraday fluctuation during the period of the loading test [10]. This is especially true if the decrease in blood Phe concentration at 
24 hour is around 30 to $40 \%$, like in two cPKU patients in this study. This causes to mis-conclude that the patient is BH4-responsive while his genotype is predicted to be unresponsive. However, only failure of long-term Phenylalanine dietary tolerance improvement would definitively confirm that the patient is unresponsive. A third reason is an interindividual variability in $\mathrm{BH} 4$ intestinal absorption or in concentration of cofactor required for PAH activity [9]. Lastly, a dual BH4-responsiveness can be due to possible variations in the protocol of the test $[12,20]$. However, since the procedure was standardized (all centers used the same $\mathrm{BH} 4$ dose, the same time of follow-up and a common definition of responsiveness), discrepancies cannot be explained here by differences in protocols. To note, recent data from the European literature [14] showed that a 48 h-loading test would allow a better recognition of late responders. Here, a 24 h-BH4 loading test was used. Some late-responsive patients may thus have been misclassified as non-responders and some genotypes left unclassified could have been assigned a responsiveness status.

\section{Conclusion}

This study describe the correlation between $P A H$ genotyping and BH4 loading test in 364. French phenylketonuria patients. We report here that 46 genotypes and five mutations, whose $\mathrm{BH} 4$-responsiveness was previously unknown, are BH4-responsive. Our observations additionally suggest that mHP patients carrying two responsive alleles are likely to be always BH4-responders. Conversely, patients with 2 non-responsive alleles as well as carriers of combinations of frameshift, in-frame, nonsense, or splicesite mutations are likely to not respond to BH4. For the other cases, the presence of at least one missense responsive mutation is necessary, but not sufficient, for the patient to be responsive. In these patients, BH4-loading test and long-term monitoring of dietary Phe tolerance are the mainstay of the evaluation of BH4-responsiveness.

This study underscores the relevance of systematic incorporation of $P A H$ genotyping together with $\mathrm{BH} 4$-loading test in the clinical evaluation of BH4-responsiveness to help distinguishing true response to $\mathrm{BH} 4$ administration.

\section{Ethics, consent and permissions}

All procedures followed were in accordance with the ethical standards of the responsible committee on human experimentation and with the Helsinki Declaration of 1975. Informed consent for genotype assessment was obtained from patients (or parents) for being included in the study.

\section{Additional files}

\begin{abstract}
Additional file 1: Figure S1. Frequency of positive $\mathrm{BH} 4$-loading test is higher in mildest PKU phenotypes. Error bars represent the $95 \%$ confidence intervals. Fisher exact test, ${ }^{* *} p \leq 0.001,{ }^{* *} p \leq 0.01,{ }^{*} p \leq 0.05$. (TIF $32 \mathrm{~kb}$ )

Additional file 2: Figure S2. The 5 mutations identified in this study as being $\mathrm{BH} 4$-responsive are localized in the catalytic domain of phenylalanine hydroxylase. The PAH monomer is drawn as a ribbon representation complexed with tetrahydrobiopterin ( $\mathrm{BH} 4$ orange balls), thienylalanine (analog substrate) and iron (red sphere). The $\mathrm{N}$-terminal regulatory domain (residues 103 - 142) is in blue, the catalytic domain (residues 143-410) is in green and the dimerization motif (residues 411-427) is in purple. Mutated aminoacids are indicated in red. (TIF $1429 \mathrm{~kb}$ )
\end{abstract}

Additional file 3: Figure S3. PAH secondary structure. Alpha-helices, beta-strands and turns across the regulatory, catalytic and oligomerization domains are indicated. Important residues are represented as well as the position of the five mutations identified in this study as being $\mathrm{BH} 4$ responsive. ARS: Autoregulatory sequence, CBR: Cofactor binding region. (TIF $315 \mathrm{~kb}$ )

Additional file 4: Figure S4. Percentage of positive $\mathrm{BH} 4$ loading tests depending on responsiveness of both alleles in the different severity groups. The diagram shows that frequency of $\mathrm{BH} 4$ positive test in $\mathrm{mHP}$ patients carrying 2 responsive alleles is $100 \%$, whereas this percentage is $85.7 \%$ in patients with $\mathrm{mPKU}$ and $42.3 \%$ in patients with cPKU. A similar trend is observed for patients carrying only $1 \mathrm{BH} 4$-responsive mutation. The 2 patients with tPKU and 2 non-responsive alleles classified as $\mathrm{BH} 4$ responders, moderately lowered the Phe concentrations (minus $31 \%$ and minus $33 \%$ ). This decrease would be clinically irrelevant as developed in the discussion and highlights the importance of combining genotyping and $\mathrm{BH} 4$ test before classifying a patient as $\mathrm{BH} 4$ responsive or not. Fisher exact test, ${ }^{* * *} p \leq 0.001,{ }^{* *} p \leq 0.01,{ }^{*} p \leq 0.05$. (TIF $44 \mathrm{~kb}$ )

Additional file 5: Figure S5. Percentage of positive BH4-loading tests depending on the type of each of the two mutations of the genotypes. Missense mutations significantly correlate with positive $\mathrm{BH} 4$ loading test more frequently than any other type of mutation. miss: missence. ns: nonsense.fs: frameshift. del: deletion. dupl: duplication. Fisher exact test, ${ }^{* * *} p \leq 0.001,{ }^{* *} p \leq 0.01,{ }^{*} p \leq 0.05$. (TIF $1173 \mathrm{~kb}$ )

Additional file 6: Figure S6. Percentage of positive $\mathrm{BH} 4$ loading tests depending on domain localization of alleles from each genotype. Fisher exact test, ${ }^{* * *} p \leq 0.001,{ }^{* *} p \leq 0.01,{ }^{*} p \leq 0.05$. (TIF $33 \mathrm{~kb}$ )

Additional file 7: Figure S7. Percent of positive $\mathrm{BH} 4$-loading tests depending on localization of missense mutations. This figure concern only patients which carry at least one missense mutation $(n=311)$. Numbers indicated in each column represent the global number of observations (both responsive and non-responsive tests) for each column. ex: exon. Fisher exact test, ${ }^{* * * *} p \leq 0.0001,{ }^{* * *} p \leq 0.001,{ }^{* *} p \leq 0.01,{ }^{*} p \leq 0.05$. (TIF $53 \mathrm{~kb}$ )

\section{Competing interests}

Elise Jeannesson-Thivisol, François Feillet, Céline Chéry, Pascal Perrin, Shyue-Fang Battaglia-Hsu, Bernard Herbeth, Aline Cano, Magalie Barth, Alain Fouilhoux, Karine Mention, François Labarthe, Jean-Baptiste Arnoux, François Maillot, Catherine Lenaerts, Cécile Dumesnil, Kathy Wagner, Daniel Terral, Pierre Broué, Loïc de Parscau, Claire Gay, Alice Kuster, Antoine Bédu, Gérard Besson, Delphine Lamireau, Sylvie Odent, Alice Masurel, Jean-Louis Guéant and Fares Namour declare that they have no competing interests.

Authors' contributions

EJT, FF, JLG, FN designed study. CC, PP, performed experiments. EJT, FF, JLG, $\mathrm{FN}, \mathrm{BH}, \mathrm{CC}$ analyzed data. EJT, SFBH, FF, JLG, FN wrote and revised manuscript. FF and all other authors not mentioned above provided samples and clinical information. All authors read and approved the final manuscript.

\section{Author details}

${ }^{1}$ Reference Center for Inherited Metabolic Diseases, University Hospital of Nancy, 9 ave Forêt de Haye, BP 184, 54511 Vandoeuvre-lès-Nancy, France. ${ }^{2}$ INSERM U954, Department of Nutrition-Genetics-Environmental Risk Exposure, University of Lorraine, 9 ave Forêt de Haye, BP 184, 54511 Vandoeuvre-lès-Nancy, France. ${ }^{3}$ Reference Center for Inherited Metabolic 
Diseases, Timone Hospital, Marseille, France. ${ }^{4}$ Department of Biochemistry and Genetics, Angers University Hospital, Angers, France. ${ }^{5}$ Reference Center for Inherited Metabolic Diseases, Hospices Civils de Lyon, Bron, France. ${ }^{6}$ Reference Center for Inherited Metabolic Diseases, Jeanne de Flandres Hospital, Lille, France. ${ }^{7}$ Department of Pediatric Medicine, Clocheville Hospital, Tours, France. ${ }^{8}$ Reference Center for Inherited Metabolic Diseases, Necker-Enfants Malades Hospital, Paris, France. 'Department of Internal Medicine, Tours University Hospital, Tours, France. ${ }^{10}$ Department of Pediatrics, Amiens University-Hospital, Amiens, France. ${ }^{11}$ Pediatric Hematology and Oncology, Rouen University-Hospital, Rouen, France. ${ }^{12}$ Department of Pediatrics, Lenval Hospital, Nice, France. ${ }^{13}$ Department of Pediatrics, Hotel-Dieu Hospital, Clermont-Ferrand, France. ${ }^{14}$ Department of Pediatric Hepatology and Metabolic Diseases, Children Hospital, Toulouse, France. ${ }^{15}$ Department of Pediatrics, CHRU Morvan, Brest, France. ${ }^{16}$ Department of Pediatrics, Saint-Etienne University-Hospital, Saint-Etienne, France. ${ }^{17}$ Pediatric Department, Nantes University Hospital, Nantes, France. ${ }^{18}$ Neonatology Department, Mère-Enfant Hospital, Limoges, France. ${ }^{19}$ Department of Neurology, University Hospital of Grenoble, Grenoble, France. ${ }^{20}$ Department of Pediatrics, Pellegrin-Enfants Hospital, Bordeaux, France. ${ }^{21}$ Department of Clinical Genetics, Rennes University Hospital, Rennes, France. ${ }^{22}$ Department of Medical Genetics, Dijon University-Hospital, Dijon, France.

\section{Received: 7 August 2015 Accepted: 8 December 2015} Published online: 15 December 2015

\section{References}

1. Scriver CR. The PAH, gene, phenylketonuria, and a paradigm shift. Hum Mutat. 2007;28(9):831-45.

2. Blau NY and Yue W. BioPKU database. http://www.biopku.org

3. Guldberg P, Rey F, Zschocke J, Romano V, Francois B, Michiels L, et al. A European multicenter study of phenylalanine hydroxylase deficiency: classification of 105 mutations and a general system for genotype-based prediction of metabolic phenotype. Am J Hum Genet. 1998;63(1):71-9.

4. Heintz C, Cotton RG, Blau N. Tetrahydrobiopterin, its mode of action on phenylalanine hydroxylase, and importance of genotypes for pharmacological therapy of phenylketonuria. Hum Mutat. 2013;34(7):927-36.

5. Feillet F, van Spronsen FJ, MacDonald A, Trefz FK, Demirkol M, Giovannini $\mathrm{M}$, et al. Challenges and pitfalls in the management of phenylketonuria. Pediatrics. 2010;126(2):333-41.

6. Levy HL, Milanowski A, Chakrapani A, Cleary M, Lee P, Trefz FK, et al. Efficacy of sapropterin dihydrochloride (tetrahydrobiopterin, $6 \mathrm{R}-\mathrm{BH} 4$ ) for reduction of phenylalanine concentration in patients with phenylketonuria: a phase III randomised placebo-controlled study. Lancet. 2007;370(9586):504-10.

7. Gersting SW, Kemter KF, Staudigl M, Messing DD, Danecka MK, Lagler FB, et al. Loss of function in phenylketonuria is caused by impaired molecular motions and conformational instability. Am J Hum Genet. 2008;83(1):5-17.

8. Pey AL, Perez B, Desviat LR, Martinez MA, Aguado C, Erlandsen $\mathrm{H}$, et al Mechanisms underlying responsiveness to tetrahydrobiopterin in mild phenylketonuria mutations. Hum Mutat. 2004;24(5):388-99.

9. Staudigl M, Gersting SW, Danecka MK, Messing DD, Woidy M, Pinkas D, et al. The interplay between genotype, metabolic state and cofactor treatment governs phenylalanine hydroxylase function and drug response. Hum Mol Genet. 2011;20(13):2628-41.

10. Zurfluh MR, Zschocke J, Lindner M, Feillet F, Chery C, Burlina A, et al. Molecular genetics of tetrahydrobiopterin-responsive phenylalanine hydroxylase deficiency. Hum Mutat. 2008;29(1):167-75.

11. Quirk ME, Dobrowolski SF, Nelson BE, Coffee B, Singh RH. Utility of phenylalanine hydroxylase genotype for tetrahydrobiopterin responsiveness classification in patients with phenylketonuria. Mol Genet Metab. 2012;107(1-2):31-6.

12. Trefz FK, Scheible D, Gotz H, Frauendienst-Egger G. Significance of genotype in tetrahydrobiopterin-responsive phenylketonuria. J Inherit Metab Dis. 2009;32(1):22-6

13. Aldamiz-Echevarria L, Bueno MA, Couce ML, Lage S, Dalmau J, Vitoria I, et al. 6R-tetrahydrobiopterin treated PKU patients below 4 years of age: Physical outcomes, nutrition and genotype. Mol Genet Metab. 2015;115(1):10-6.

14. Anjema K, van Rijn M, Hofstede FC, Bosch AM, Hollak CE, Rubio-Gozalbo E, et al. Tetrahydrobiopterin responsiveness in phenylketonuria: prediction with the 48-hour loading test and genotype. Orphanet J Rare Dis. 2013;8:103.
15. Couce ML, Boveda MD, Fernandez-Marmiesse A, Miras A, Perez B, Desviat LR, et al. Molecular epidemiology and BH4-responsiveness in patients with phenylalanine hydroxylase deficiency from Galicia region of Spain. Gene. 2013;521(1):100-4.

16. Hennermann JB, Roloff S, Gebauer C, Vetter B, von Arnim-Baas A, Monch E. Long-term treatment with tetrahydrobiopterin in phenylketonuria: treatment strategies and prediction of long-term responders. Mol Genet Metab. 2012;107(3):294-301.

17. Nielsen JB, Nielsen KE, Guttler F. Tetrahydrobiopterin responsiveness after extended loading test of 12 Danish PKU patients with the Y414C mutation. J Inherit Metab Dis. 2010;33(1):9-16.

18. Scala I, Concolino D, Della Casa R, Nastasi A, Ungaro C, Paladino S, et al. Long-term follow-up of patients with phenylketonuria treated with tetrahydrobiopterin: a seven years experience. Orphanet J Rare Dis. 2015;10:14.

19. Tao J, Li N, Jia H, Liu Z, Li X, Song J et al. Correlation between genotype and the tetrahydrobiopterin-responsive phenotype in Chinese patients with phenylketonuria. Pediatr Res. 2015. doi:10.1038/pr.2015.167. [Epub ahead of print].

20. Utz JR, Lorentz CP, Markowitz D, Rudser KD, Diethelm-Okita B, Erickson D, et al. START, a double blind, placebo-controlled pharmacogenetic test of responsiveness to sapropterin dihydrochloride in phenylketonuria patients. Mol Genet Metab. 2012;105(2):193-7.

21. Blau N, van Spronsen FJ, Levy HL. Phenylketonuria Lancet. 2010; 376(9750):1417-27.

22. HAS. Plan National de Diagnotic et de Soins pour la Phénylcétonurie. 2010. http://www.has-sante.fr/portail/upload/docs/application/pdf/2010-05/ald_ 17_pnds_pcu_web.pdf.

23. Brautigam S, Kujat A, Kirst P, Seidel J, Luleyap HU, Froster UG. DHPLC mutation analysis of phenylketonuria. Mol Genet Metab. 2003;78(3):205-10.

24. National Center for Biotechnology Information. NCBI database. http://www. ncbi.nlm.nih.gov.

25. Erlandsen H, Patch MG, Gamez A, Straub M, Stevens RC. Structural studies on phenylalanine hydroxylase and implications toward understanding and treating phenylketonuria. Pediatrics. 2003;112(6 Pt 2):1557-65.

26. Camp KM, Parisi MA, Acosta PB, Berry GT, Bilder DA, Blau N, et al. Phenylketonuria Scientific Review Conference: state of the science and future research needs. Mol Genet Metab. 2014;112(2):87-122.

27. AFDPHE. Bilan d'activité 2013. http://www.afdphe.org/sites/default/files/ bilan_activite_2013.pdf.

28. Ho G, Alexander I, Bhattacharya K, Dennison B, Ellaway C, Thompson S, et al. The Molecular Bases of Phenylketonuria (PKU) in New South Wales, Australia: Mutation Profile and Correlation with Tetrahydrobiopterin (BH4) Responsiveness. JIMD Rep. 2014;14:55-65.

29. Sterl E, Paul K, Paschke E, Zschocke J, Brunner-Krainz M, Windisch E, et al. Prevalence of tetrahydrobiopterine ( $\mathrm{BH} 4)$-responsive alleles among Austrian patients with PAH deficiency: comprehensive results from molecular analysis in 147 patients. J Inherit Metab Dis. 2013;36(1):7-13.

30. Dobrowolski SF, Heintz C, Miller T, Ellingson C, Ellingson C, Ozer I, et al. Molecular genetics and impact of residual in vitro phenylalanine hydroxylase activity on tetrahydrobiopterin responsiveness in Turkish PKU population. Mol Genet Metab. 2011;102(2):116-21.

31. Heintz C, Dobrowolski SF, Andersen HS, Demirkol M, Blau N, Andresen BS. Splicing of phenylalanine hydroxylase (PAH) exon 11 is vulnerable: molecular pathology of mutations in PAH exon 11. Mol Genet Metab. 2012;106(4):403-11.

32. Song F, Qu YJ, Zhang T, Jin YW, Wang H, Zheng XY. Phenylketonuria mutations in Northern China. Mol Genet Metab. 2005;86 Suppl 1:S107-18.

33. Jennings $I G$, Cotton $R G$, Kobe B. Functional analysis, using in vitro mutagenesis, of amino acids located in the phenylalanine hydroxylase active site. Arch Biochem Biophys. 2000;384(2):238-44.

34. Danecka MK, Woidy M, Zschocke J, Feillet F, Muntau AC, Gersting SW. Mapping the functional landscape of frequent phenylalanine hydroxylase (PAH) genotypes promotes personalised medicine in phenylketonuria. J Med Genet. 2015;52(3):175-85.

35. Daniele A, Scala I, Cardillo G, Pennino C, Ungaro C, Sibilio M, et al. Functional and structural characterization of novel mutations and genotype-phenotype correlation in 51 phenylalanine hydroxylase deficient families from Southern Italy. FEBS J. 2009;276(7):2048-59.

36. Jennings IG, Cotton RG, Kobe B. Structural interpretation of mutations in phenylalanine hydroxylase protein aids in identifying genotype-phenotype correlations in phenylketonuria. Eur J Hum Genet. 2000;8(9):683-96. 